\title{
Distinguishing Vagueness from Ambiguity in Rough Set Approximations
}

\author{
Salvatore Greco \\ Department of Economics and Business, University of Catania, 95129 Catania, Italy \\ Portsmouth Business School, Centre of Operations Research and Logistics (CORL), \\ University of Portsmouth, Portsmouth, PO1 3DE United Kingdom \\ Benedetto Matarazzo \\ Department of Economics and Business, University of Catania, 95129 Catania, Italy \\ Department of Law, LUMSA University, Palermo, Italy \\ Roman Słowiński \\ Institute of Computing Science, Poznań University of Technology, 60-965 Poznań, Poland \\ Institute for Systems Research, Polish Academy of Sciences, 01-447 Warsaw, Poland
}

In this paper we present a new approach to rough set approximations that permits to distinguish between two kinds of "imperfect" knowledge in a joint framework: on one hand, vagueness, due to imprecise knowledge and uncertainty typical of fuzzy sets, and on the other hand, ambiguity, due to granularity of knowledge originating from the coarseness typical of rough sets. The basic idea of our approach is that each concept is represented by an orthopair, that is, a pair of disjoint sets in the universe of knowledge. The first set in the pair contains all the objects that are considered as surely belonging to the concept, while the second set contains all the objects that surely do not belong to the concept. In this context, following some previous research conducted by us on the algebra of rough sets, we propose to define as rough approximation of the orthopair representing the considered concept another orthopair composed of lower approximations of the two sets in the first orthopair. We shall apply this idea to the classical rough set approach based on indiscernibility, as well as to the dominance-based rough set approach. We discuss also a variable precision rough approximation, and a fuzzy rough approximation of the orthopairs. Some didactic examples illustrate the proposed methodology.

Keywords: Imperfect knowledge, Vagueness, Ambiguity, Rough sets, Dominance-based Rough Set Approach, Pawlak operator, Fuzzy rough approximations, Variable precision rough approximations

\section{Introduction}

Vagueness and ambiguity are concepts that after attracting the interest of philosophers, gain more and more attention from researchers in computational intelligence, 
where they are used to distinguish between two types of imperfect knowledge. In computational intelligence, vagueness is dealt by fuzzy set theory, while ambiguity caused by coarseness of information by rough set theory. ${ }^{1,2}$ This distinction has been acknowledged by the following opinion of Dubois and Prade: "Rough sets have often been compared to fuzzy sets, sometimes with a view to introduce them as competing models of imperfect knowledge. Such a comparison is misfounded. Indiscernibility and vagueness are distinct facets of imperfect knowledge. Indiscernibility refers to the granularity of knowledge (classes of indiscernible objects). Vagueness is due to the fact that terms of natural language are often gradual notions, and refer to sets with smooth boundaries. Fuzzy set theory relies on ordering relations that express intensity of membership. Rough set theory is based on equivalence relations describing partitions made of classes of indiscernible objects. So, one theory is quite distinct from the other, and they display a natural complementarity. And it would be nice to use both of them conjointly, since vagueness and coarseness are sometimes interacting".

A conjoint use of fuzzy sets and rough sets has been often discussed and investigated since the beginning of nineties, taking as a staring point the rough set concepts which were then reformulated in a fuzzy context by means of fuzzy logical connectives (see, e.g. ${ }^{4,5}$ ). In this paper, we want to reconsider the distinction between vagueness and ambiguity, but from a more fundamental point of view, having in mind the following meaning of the two types of imperfect knowledge:

- vagueness, related to 'a priori' epistemic evaluation of the credibility of one concept, and

- ambiguity, related to 'a posteriori' approximation of the same concept by means of granules of knowledge.

Our approach is rooted in a general conception of imprecision due to Frege who expressed the idea of imprecision in the following way: ${ }^{6}$

"The concept must have a sharp boundary. To the concept without a sharp boundary there would correspond an area that had not a sharp boundary-line all around."

The Frege's idea of imprecision introducing boundary line cases that can be assigned neither to the concept nor to its complement was an inspiration for Pawlak and a philosophical basis of rough set theory $\left(\mathrm{see}^{7,8}\right)$.

A natural and straightforward way of representing the imprecision is to assign to each concept $\mathcal{C}$ a pair of disjoint sets $(X, Y)$ of the universe of discourse $U$, technically called orthopairs, ${ }^{9}$ such that $X$ contains the objects that for sure belong to $\mathcal{C}$ and $Y$ contains all the objects that for sure do not belong to $\mathcal{C}$. This idea has been considered in many theories related to the handling of uncertainty, such as three valued logic, ${ }^{10}$ shadowed sets,${ }^{11}$ or quantum logic algebra. ${ }^{12}$ Another way to represent uncertainty, consistent with rough set theory, is to use the interval set ${ }^{13}$ defined as a pair of sets $(W, Z), W \subseteq Z \subseteq U$, such that $W$ contains all the objects that surely belong to $\mathcal{C}$ and $Z$ contains the objects that could belong to 
$\mathcal{C}$. Of course, there is a clear correspondence between orthopairs and interval sets. In fact, the objects that could belong to concept $\mathcal{C}$ are those that remain in the universe once the objects that for sure do not belong to $\mathcal{C}$ are removed, so that the orthopair $(X, Y)$ is equivalent to the interval set $(X, U-Y)$. Therefore, for the moment we shall focus our attention on orthopairs, but it is clear that what is said for orthopairs can be extended to intervals sets. Later in the paper, we shall come back to interval sets pointing out how the concepts introduced for orthopairs can be generalized to interval sets.

The idea of representing imprecision using orthopairs has been exploited in the domain of algebraic theory for imprecise concepts. Among these works a special place has been taken by an algebraic approach to rough set theory ${ }^{14}$ where pairs $(X, Y)$ of disjoint sets in a universe of discourse $U$, that is, orthopairs, are considered. In the orthopair, set $X$ is the lower approximation (interior) of a given set $Z \subseteq U$, and $Y$ is the complement of the upper approximation (exterior) of set $Z$. Let us remember that the lower approximation $X$ of a given set $Z$ is the set of all the objects that not only belong to $Z$ but, moreover, according to the available information, all objects indiscernible with them also belong to $Z$. For example, student $S$ belongs to the lower approximation $\underline{G}$ of class $G$ of good students not only if he belongs to $G$, but also if all students having the same evaluations on all considered subjects belong to the same class.

In this paper, we propose a different model of rough set theory. We take into account a pair of disjoint sets to represent an a priori vagueness such that, given concept $\mathcal{C}$, the pair $(X, Y)$ is composed of set $X$ being the necessity kernel of the objects that for sure belong to concept $\mathcal{C}$, and set $Y$ being the non-possibility kernel of the objects that for sure do not belong to concept $\mathcal{C}$. To take into account an a posteriori ambiguity related to the granularity of knowledge about concept $\mathcal{C}$, we assign the pair $(\underline{X}, \underline{Y})$ to the pair $(X, Y)$. This permits to distinguish between vagueness understood as imprecise or uncertain knowledge represented typically by fuzzy sets of the original pair $(X, Y)$, on one hand, and ambiguity, caused by indiscernibility and coarseness of the granular information, represented typically by rough sets of the derived pair $(\underline{X}, \underline{Y})$, on the other hand.

For example, as the concept 'good' students is vague, instead of considering a precise class of good students, one can consider a pair $\left(G^{P}, G^{N}\right)$ of sets of students, where

- $G^{P}$ contains the students that are considered 'good', and

- $G^{N}$ contains the students that are considered 'not good'.

After, taking into account the evaluations of students on the subjects considered relevant for assessing their quality, one can assign the pair $\left(\underline{G}^{P}, \underline{G}^{N}\right)$ to the pair $\left(G^{P}, G^{N}\right)$, such that

- $\underline{G}^{P}$ contains the students that, taking into account the available informa- 
tion, are considered 'good' without any doubt, and

- $\underline{G}^{N}$ contains the students that, taking into account the available information, are considered 'not good' without any doubt.

Let us observe that in the example of students, when defining the lower approximations, we have not taken into account the ordinal properties of the assignment to the sets of 'good' and 'not good' students, and of the evaluations on the subjects considered relevant. Thus, we considered the set qualifiers 'good' and 'not good', as well as different evaluations on the subjects, e.g., 'medium' or 'bad', as different labels. Therefore, student $S$ belonging to the set of 'good' students $G^{P}$ cannot be assigned to the lower approximation $\underline{G}^{P}$ of the same set when another student $S^{\prime}$ not belonging to $G^{P}$ has the same evaluations on all the subjects considered relevant. Indeed, the indiscerniblity principle adopted to define $\left(\underline{G}^{P}, \underline{G}^{N}\right)$ says that all objects (students) having the same evaluations on all the relevant attributes (subjects) should have been assigned to the same class and, if this did not happen, there is an ambiguity, and the corresponding objects (students) cannot be assigned to the lower approximations of their classes.

If we would take into account the ordinal properties of the above set qualifiers and evaluations, then 'good' should be considered as better than 'not good', as well as 'medium' should be considered as better than 'bad'. In fact, one could have two objects (students), $S$ and $S^{\prime}$, such that $S$ has been assigned to a better class than $S^{\prime}$ (for example, $S$ assigned to the class of 'good' students and $S^{\prime}$ assigned to the class of 'not good' students), while having different evaluations on the relevant attributes (subjects). As they are discernible with respect to the available information they are assigned to the lower approximations of their respective classes and this assignment does not violate the indiscernibility principle. However, it could happen that $S$, even if assigned to a better class, has not better evaluations than $S^{\prime}$ on all the relevant attributes, so that, despite their discernibility, the assignment of $S$ and $S^{\prime}$ to the lower approximations of their respective classes would appear ambiguous. In fact, in this case, one would expect to apply the dominance principle according to which if an object (student) has not worse evaluations than another object (student) on all the relevant attributes (subjects), then the first object (student) should belong to a class not worse than that of the second object (student), and, if this does not happen, there is an ambiguity and the corresponding objects (students) cannot be assigned to the lower approximations of their classes.

This example leads to a conclusion that when the ordinal properties of set qualifiers (classes) and evaluations are taken into account, the classical rough set approximations, considered within the Indiscernibility-based Rough Set Approach (IRSA), have to be redefined by replacing the indiscernibility relation with the dominance relation. The latter relates two objects (students) $S$ and $S^{\prime}$ when $S$ has not worse evaluations than $S^{\prime}$ on all the relevant attributes (subjects). This is the perspective of the Dominance-based Rough Set Approach (DRSA ${ }^{15-17}$ ) which associates the pair $\left(\underline{X}^{\uparrow}, \underline{Y}^{\downarrow}\right)$ to the pair $(X, Y)$, such that 
- $\underline{X}^{\uparrow}$ is the upward lower approximation of $X$, that is, the set of all the objects that not only belong to $X$ but, moreover, according to available information, all objects dominating them also belong to $X$,

- $\underline{Y}^{\downarrow}$ is the downward lower approximation of $Y$, that is, the set of all the objects that not only belong to $Y$, but, moreover, according to available information, all objects dominated by them also belong to $Y$.

Returning to our example with the students, let us observe that, taking into account the available information about evaluations of students on the subjects considered relevant, one can assign the pair $\left(\underline{G}^{P \uparrow}, \underline{G}^{N \downarrow}\right)$ to the pair $\left(G^{P}, G^{N}\right)$, such that

- $\underline{G}^{P \uparrow}$ contains the students that are considered 'good' without any doubt, in the sense that not only they belong to $G^{P}$, but also all the students having not worse evaluation than them on all the subjects, i.e., dominating them, belong to $G^{P}$, and

- $\underline{G}^{N \downarrow}$ the students that are considered 'not good' without any doubt, in the sense that not only they belong to $G^{N}$, but also all the students having not better evaluation than them on all the subjects, i.e., dominated by them, belong to $G^{N}$.

The operator assigning the pair $(\underline{X}, \underline{Y})$ to the pair $(X, Y)$ has been called Pawlak operator in, ${ }^{18}$ where it has been proposed and investigated as a new algebraic model for rough set theory (for a survey on algebraic structures for rough set theory see chapter $\left.8 \mathrm{in}^{19}\right)$. The Pawlak operator assigning the pair $\left(\underline{X}^{\uparrow}, \underline{Y}^{\downarrow}\right)$ to the pair $(X, Y)$ has been later considered $i^{20}$ as an abstract algebraic model for DRSA. In this paper, we come back to the above considerations, defining a new rough set approach that permits to distinguish vagueness of the evaluations, represented by $(X, Y)$, from the ambiguity due to coarseness of available knowledge, represented by $(\underline{X}, \underline{Y})$ and $\left(\underline{X}^{\uparrow}, \underline{Y}^{\downarrow}\right)$. Our aim is to redefine these fundamental concepts of imperfect knowledge within the framework of rough set theory, and to investigate basic properties of rough approximations in this context.

The paper is organized as follows. Next section recalls the basic concepts of the rough set approach based on indiscernibility. Section 3 presents the rough approximations $(\underline{X}, \underline{Y})$ of orthopairs $(X, Y)$ in terms of indiscernibility relations. Section 4 recalls Dominance-based Rough Set Approach, and section 5 introduces rough approximations $\left(\underline{X}^{\uparrow}, \underline{Y}^{\downarrow}\right)$ of orthopairs $(X, Y)$ based on dominance relations. In section 6 , some extensions related to generalizations of rough sets, such as fuzzy rough approximations and variable precision rough approximations, are discussed. Section 7 collects conclusions and outlines future developments.

\section{Basic concepts of rough set approximations}

An information table $\mathcal{I} \mathcal{T}$ stores data on objects of a universe of discourse $U$ described by some attributes of interest. Rows of $\mathcal{I} \mathcal{T}$ refer to distinct objects, while 
columns refer to different attributes, so that that each cell of the table indicates an evaluation (quantitative or qualitative) of the object placed in the corresponding row by means of the attribute from the corresponding column. In general, an information table is the 4-tuple $\mathcal{I} \mathcal{T}=\langle U, Q, V, v\rangle$, where $U$ is a finite non-empty set of objects, called universe, $Q=\left\{q_{1}, \ldots, q_{n}\right\}$ is a finite set of attributes, $V_{q}$ is a value set of the attribute $q, V=\bigcup_{q \in Q} V_{q}$, and $v: U \times Q \rightarrow V$ is a total function such that $v(x, q) \in V_{q}$ for each $q \in Q, x \in U$, called information function.

To every (non-empty) subset of attributes $P \subseteq Q$ there is associated an indiscernibility relation on $U$, denoted by $I_{P}$ :

$$
I_{P}=\{(x, y) \in U \times U: v(x, q)=v(y, q), \text { for all } q \in P\} .
$$

Very often we shall write $x I_{P} y$ instead of $(x, y) \in I_{P}$. If $(x, y) \in I_{P}$, it is said that the objects $x$ and $y$ are $P$-indiscernible. In the sequel we will simply write the objects $x$ and $y$ are indiscernible, unless this causes an ambiguity. Clearly, the indiscernibility relation thus defined is an equivalence relation (reflexive, symmetric and transitive).

The equivalence class of any $x \in U$ with respect to indiscernibility relation $I_{P}$, $\emptyset \subset P \subseteq Q$,

$$
I_{P}(x)=\left\{y \in U: y I_{P} x\right\}
$$

is called $P$-elementary set.

Given $P \subseteq Q$ and $X \subseteq U$, using the $P$-elementary sets, the $P$-lower and the $P$-upper approximation of $X$ are defined, respectively, as follows:

$$
\begin{gathered}
\underline{I}_{P}(X)=\bigcup_{I_{P}(x) \subseteq X} I_{P}(x), \\
\bar{I}_{P}(X)=\bigcup_{I_{P}(x) \cap X \neq \emptyset} I_{P}(x) .
\end{gathered}
$$

The $P$-lower and the $P$-upper approximation of $X, \underline{I}_{P}(X)$ and $\bar{I}_{P}(X)$, can be also formulated as follows:

$$
\begin{gathered}
\underline{I}_{P}(X)=\left\{x \in U: I_{P}(x) \subseteq X\right\}, \\
\bar{I}_{P}(X)=\left\{x \in U: I_{P}(x) \cap X \neq \emptyset\right\} .
\end{gathered}
$$

Set $B n_{P}(X)=\bar{I}_{P}(X)-\underline{I}_{P}(X)$ is called the $P$-boundary of $X$. Taking into account the information provided by attributes from $P$ only, the set $\underline{I}_{P}(X)$ is the set of objects from $U$ which can be certainly classified as elements of $X$, and the set $\bar{I}_{P}(X)$ is the set of objects from $U$ which can be possibly classified as elements of $X$. The set $B n_{P}(X)$ contains objects from $U$ which cannot be classified with certainty as belonging either to $X$ or to its complement, taking into account the information provided by attributes from $P$ only. The objects from $B n_{P}(X)$ are said to be $P$-inconsistent with respect to $X$, while other objects from $U$ are said to be 
$P$-consistent with respect to $X$. The ratio of the number of objects included in the $P$-lower approximation of $X$ to the number of objects assigned to $X$ is called quality of approximation of set $X$ by attributes from $P$ :

$$
\gamma_{P}(X)=\frac{\left|\underline{I}_{P}(X)\right|}{|X|} .
$$

Given $X \subseteq U$, using the information provided by attributes from $P \subseteq Q$, we are able to define two regions of certainty, called $P$-positive region and $P$-negative region, respectively:

$$
\begin{gathered}
\operatorname{Pos}_{P}(X)=\underline{I}_{P}(X), \\
\operatorname{Neg}_{P}(X)=U-\bar{I}_{P}(X) .
\end{gathered}
$$

In fact, using information provided by attributes from $P$, objects from $\operatorname{Pos}_{P}(X)$ can be classified with complete certainty as belonging to $X$, while objects from $N e g_{P}(X)$ can be classified with complete certainty as not belonging to $X$.

For a particular object $x \in U$, it is interesting to use the available information to assess the degree of its membership in a subset $X$ of $U$. In this perspective, given $P \subseteq Q$ and $X \subseteq U$, the degree of membership of $x$ in $X$ can be defined as

$$
\mu_{X}^{P}(x)=\frac{\left|X \cap I_{P}(x)\right|}{\left|I_{P}(x)\right|} .
$$

$\mu_{X}^{P}(x)$ is called rough membership function.

The value of $\mu_{X}^{P}(x)$ may be interpreted as a conditional probability of the membership in $X$ given the membership in $I_{P}(x) \cdot \mu_{X}^{P}(x)$ may also be understood as the degree of certainty (credibility) to which $x$ belongs to $X$. Observe that the value of the membership function is calculated from the available data, and not subjectively assumed, as it is often the case of membership functions of fuzzy sets.

The lower and upper approximations satisfy the following properties.

Theorem 1.1,2 For any $X, Y \subseteq U$ and for any $P \subseteq Q$,

(1) $\underline{I}_{P}(X) \subseteq X \subseteq \bar{I}_{P}(X)$,

(2) $\underline{I}_{P}(\emptyset)=\bar{I}_{P}(\emptyset)=\emptyset, \underline{I}_{P}(U)=\bar{I}_{P}(U)=U$,

(3) $\bar{I}_{P}(X \cup Y)=\bar{I}_{P}(X) \cup \bar{I}_{P}(Y)$,

(4) $\underline{I}_{P}(X \cap Y)=\underline{I}_{P}(X) \cap \underline{I}_{P}(Y)$,

(5) $X \subseteq Y \Rightarrow \underline{I}_{P}(X) \subseteq \underline{I}_{P}(Y)$,

(6) $X \subseteq Y \Rightarrow \bar{I}_{P}(X) \subseteq \bar{I}_{P}(Y)$,

(7) $\underline{I}_{P}(X \cup Y) \supseteq \underline{I}_{P}(X) \cup \underline{I}_{P}(Y)$,

(8) $\bar{I}_{P}(X \cap Y) \subseteq \bar{I}_{P}(X) \cap \bar{I}_{P}(Y)$,

(9) $\underline{I}_{P}(U-X)=U-\bar{I}_{P}(X)$,

(10) $\bar{I}_{P}(U-X)=U-\underline{I}_{P}(X)$,

(11) $\underline{I}_{P}\left[\underline{I}_{P}(X)\right]=\bar{I}_{P}\left[\underline{I}_{P}(X)\right]=\underline{I}_{P}(X)$,

(12) $\bar{I}_{P}\left[\bar{I}_{P}(X)\right]=\underline{I}_{P}\left[\bar{I}_{P}(X)\right]=\bar{I}_{P}(Y)$. 
An issue of great practical importance is the reduction of "superfluous" attributes in an information table. Superfluous attributes can be eliminated without deteriorating the information contained in the original table.

Let $P \subseteq Q$ and $p \in P$. It is said that attribute $p$ is superfluous in $P$ with respect to approximation of $X \subseteq U$ if $\underline{I}_{P}(X)=\underline{I}_{P-\{p\}}(X)$; otherwise, $p$ is indispensable in $P$. The subset of $Q$ containing all the indispensable attributes is known as the core. Any minimal (with respect to inclusion) subset $P \subseteq Q$, such that $\underline{P}(X)=Q(X)$, is called reduct with respect to the approximation of $X$. It specifies a minimal subset $P$ of $Q$ which keeps the quality of approximation of set $X$ at the same level as the whole set of attributes, i.e., $\gamma_{P}(X)=\gamma_{Q}(X)$. In other words, the attributes that do not belong to the reduct are superfluous with respect to the approximation of $X$. More than one reduct may exist in an information table and their intersection gives the core.

Functional dependencies between evaluations $v(x, q)$ given by attributes $q \in Q$ to objects $x \in U$ on one hand, and the membership of these objects to a target set $X \subseteq U$ on the other hand, can be usefully described in terms of decision rules. These are logical statements of the type "if..., then...", where the premise (condition part) specifies values $v(x, q)$ assumed by attributes from $P \subseteq Q$ (description of $P$-elementary sets) and the conclusion (decision part) specifies an assignment to a concept $\mathcal{C}$ represented by set $X \subseteq U$. More formally, for $X \subseteq U$ the syntax of a rule is:

"if $v\left(x, q_{1}\right)=r_{q 1}$ and $v\left(x, q_{2}\right)=r_{q 2}$ and $\ldots v\left(x, q_{p}\right)=r_{q p}$,

then $x \in X^{\prime \prime}$,

where $\left\{q_{1}, q_{2}, \ldots, q_{p}\right\} \subseteq Q,\left(r_{q 1}, r_{q 2}, \ldots, r_{q p}\right) \in V_{q 1} \times V_{q 2} \times \ldots \times V_{q p}$.

The rough approximations $\underline{I}_{P}(X)$ and $I_{P}(X)$ are very useful to induce decision rules. Indeed, procedures for generation of decision rules use an inductive learning principle. The objects are considered as examples of classification. The decision rules suggest a certain assignment to $X$ if they are induced under a hypothesis that objects belonging to $\underline{I}_{Q}(X)$ are positive examples, and all the others are negative. Instead, the decision rules suggest a possible assignment to $X$ if they are induced under a hypothesis that objects belonging to $\bar{I}_{Q}(X)$ are positive examples, and all the others are negative. Therefore, the following two types of decision rules can be considered:

1) certain decision rules, providing profile descriptions for objects belonging to $\underline{I}_{P}(X)$ :

if $f_{q_{1}}(x)=r_{q_{1}}$ and $\ldots$ and $f_{q_{p}}(x)=r_{q_{p}}$, then $x \in X$,

$\left\{q_{1}, \ldots, q_{p}\right\} \subseteq Q, r_{q_{1}}, \ldots, r_{q_{p}} \in \Re$

2) possible decision rules, providing profile descriptions for objects belonging to $\bar{I}_{P}(X)$ :

if $f_{q_{1}}(x)=r_{q_{1}}$ and $\ldots$ and $f_{q_{p}}(x)=r_{q_{p}}$, then $x$ possibly belongs to $X$, 


$$
\left\{q_{1}, \ldots, q_{p}\right\} \subseteq Q, r_{q_{1}}, \ldots, r_{q_{p}} \in \Re .
$$

For the sake of simplicity, in all the following illustrative examples we consider certain decision rules only.

An object $x \in U$ supports decision rule $r$ if its description is matching both the condition part and the decision part of the rule. We also say that decision rule $r$ covers object $x$ if it matches the condition part of the rule. Each decision rule is characterized by its strength, defined as the number of objects supporting the rule.

A decision rule is called minimal if removing any attribute from the condition part gives a rule covering also negative examples. A minimal set of rules is a family $\mathcal{R}$ of rules covering all positive examples, and such that removing a rule from $\mathcal{R}$ makes some positive example not covered.

The existing induction algorithms use one of the following strategies: ${ }^{21}$

(a) generation of a minimal representation, i.e., minimal set of rules covering all positive examples,

(b) generation of an exhaustive representation, i.e., all rules covering the set of positive examples,

(c) generation of a characteristic representation, i.e., a set of rules covering relatively many positive examples, however, not necessarily all of them.

\subsection{A didactic example}

The committee of an engineering school has to decide which candidates to accept or reject from among students applying for admission. The committee takes into consideration high school grades in Mathematics, Physics, Literature and Philosophy. The committee would like to make decisions on the basis of some requirements expressed in terms of "if ..., then ..." rules. To define these rules the committee supplies first some examples of classification decisions shown in Table 1. The evaluations of students on Mathematics, Physics, Literature and Philosophy form an information table. It is a base for approximation of the sets of accepted and rejected students.

With respect to approximation of the set of accepted students, there are two reducts:

- $\operatorname{Red}_{1}=\{$ Mathematics, Physics, Philosophy $\}$,

- $\operatorname{Red}_{2}=\{$ Physics, Literature, Philosophy $\}$.

Indeed, considering all the attributes, as well as the attributes from $\operatorname{Red}_{1}$ or from $\operatorname{Red}_{2}$, there is no case of ambiguity, that is, no set of students indiscernible with respect to one of these sets of attributes, i.e., students having the same evaluations on the subjects from the complete set or on the subjects from any reduct, have mixed and, consequently, ambiguous admission decisions. This means that the attributes "Mathematics" and "Literature" are dispensable, because each of them can be removed from the complete set of attributes (obtaining $R e d_{2}$ and $\operatorname{Red}_{1}$, 
Table 1. Exemples of classification decisions about admission of candidate students

\begin{tabular}{|c|c|c|c|c|c|}
\hline Student & Mathematics & Physics & Literature & Philosophy & Acceptance/rejection \\
\hline$S 1$ & good & good & bad & good & accepted \\
\hline$S 2$ & good & good & good & medium & accepted \\
\hline$S 3$ & medium & good & medium & good & accepted \\
\hline$S 4$ & medium & medium & medium & bad & accepted \\
\hline$S 5$ & bad & medium & medium & good & rejected \\
\hline$S 6$ & medium & medium & medium & good & rejected \\
\hline$S 7$ & bad & medium & good & good & rejected \\
\hline$S 8$ & good & medium & bad & good & accepted \\
\hline$S 9$ & medium & medium & medium & good & rejeced \\
\hline$S 10$ & medium & medium & medium & medium & accepted \\
\hline$S 11$ & bad & medium & good & medium & rejected \\
\hline$S 12$ & medium & good & bad & bad & rejected \\
\hline$S 13$ & good & bad & medium & medium & rejected \\
\hline$S 14$ & bad & good & medium & medium & rejected \\
\hline$S 15$ & bad & bad & good & medium & rejected \\
\hline
\end{tabular}

respectively), while keeping the same lower approximation of the set of accepted students. No other attribute can be further removed from $\operatorname{Red}_{1}$ and $\operatorname{Red}_{2}$ because then some case of ambiguity appears. For example, removing "Mathematics" from $\operatorname{Red}_{1}$, students $S 2$ and $S 15$ become indiscernible (both of them have an evaluation "medium" in "Literature" and "good" in "Philosophy"), but $S 2$ is accepted and $S 15$ is rejected. Therefore, $\operatorname{Red}_{1}$ and $\operatorname{Red}_{2}$ are minimal sets of attributes keeping the same lower approximation of the set of accepted students and so they are reducts. Removing instead the attribute "Physics" from the complete set, i.e., considering the set of attributes \{Mathematics, Literature, Philosophy\}, students $S 3, S 6$ and $S 9$ are indiscernible because all of them have evaluation "medium" in "Mathematics" and "Literature" and "good" in "Philosophy". However, student $S 3$ is accepted, while students $S 6$ and $S 9$ are rejected. Consequently, the attribute "Physics" is indispensable and the set of attributes \{Mathematics, Literature, Philosophy is not a reduct. Analogously, removing the attribute "Philosophy", students $S 4, S 9$ and $S 10$ are indiscernible because all of them have evaluation "medium" in "Mathematics", "Physics" and "Literature". However, student $S 4$ and $S 10$ are accepted, while student $S 9$ is rejected. Consequently, the attribute "Philosophy" is indispensable and the set of attributes \{Mathematics, Physics, Literature\} is not a reduct. The two indispensable attributes "Physics" and "Philosophy" constitute the core. Notice that $\{$ Physics, Philosophy\} is also the intersection of the two reducts Red 1 and $\operatorname{Red}_{2}$. 
The following certain decision rules have been induced from Table 1 (between braces there are id's of students supporting the corresponding rule):

rule 1) if the evaluation on Mathematics is good, and the evaluation on Physics is good, then the student is accepted, $\{S 1, S 2\}$,

rule 2) if the evaluation on Mathematics is good, and the evaluation on Physics is medium, then the student is accepted, $\{S 8\}$,

rule 3) if the evaluation on Mathematics is good, and the evaluation on Literature is bad, then the student is accepted, $\{S 1, S 8\}$,

rule 4) if the evaluation on Mathematics is good, and the evaluation on Literature is good, then the student is accepted, $\{S 2\}$,

rule 5) if the evaluation on Physics is good, and the evaluation on Literature is good, then the student is accepted, $\{S 2\}$,

rule 6) if the evaluation on Physics is medium, and the evaluation on Literature is bad, then the student is accepted, $\{S 8\}$,

rule 7) if the evaluation on Mathematics is medium, and the evaluation on Physics is good, and the evaluation on Literature is medium, then the student is accepted, $\{S 3\}$,

rule 8) if the evaluation on Mathematics is good, and the evaluation on Philosophy is good, then the student is accepted, $\{S 1, S 8\}$,

rule 9) if the evaluation on Mathematics is medium, and the evaluation on Philosophy is medium, then the student is accepted, $\{S 10\}$,

rule 10) if the evaluation on Physics is good, and the evaluation on Philosophy is good, then the student is accepted, $\{S 1, S 3\}$,

rule 11) if the evaluation on Physics is medium, and the evaluation on Philosophy is bad, then the student is accepted, $\{S 4\}$,

rule 12) if the evaluation on Literature is bad, and the evaluation on Philosophy is good, then the student is accepted, $\{S 1, S 8\}$,

rule 13) if the evaluation on Literature is medium, and the evaluation on Philosophy is bad, then the student is accepted, $\{S 4\}$,

rule 14) if the evaluation on Physics is medium, and the evaluation on Literature is medium, and the evaluation on Philosophy is medium, then the student is accepted, $\{S 10\}$,

rule 15) if the evaluation on Mathematics is bad, then the student is rejected, $\{S 5, S 7, S 11, S 14, S 15\}$,

rule 16) if the evaluation on Physics is bad, then the student is rejected, $\{S 13, S 15\}$,

rule 17) if the evaluation on Mathematics is medium, and the evaluation on Lit- 
erature is bad, then the student is rejected, $\{S 12\}$,

rule 18) if the evaluation on Mathematics is good, and the evaluation on Literature is medium, then the student is rejected, $\{S 13\}$,

rule 19) if the evaluation on Physics is medium, and the evaluation on

Literature

is good, then the student is rejected, $\quad\{S 7, S 11\}$,

rule 20) if the evaluation on Physics is good, and the evaluation on Philosophy is bad, then the student is rejected, $\{S 12\}$,

rule 21) if the evaluation on Mathematics is medium, and the evaluation on Physics is medium, and the evaluation on Philosophy is good, then the student is rejected, $\{S 6, S 9\}$,

rule 22) if the evaluation on Literature is good, and the evaluation on Philosophy is good, then the student is rejected, $\{S 7\}$,

rule 23) if the evaluation on Literature is bad, and the evaluation on Philosophy is bad, then the student is rejected, $\{S 12\}$,

rule 24) if the evaluation on Physics is medium, and the evaluation on Literature is medium, and the evaluation on Philosophy is good, then the student is rejected, $\{S 5, S 6, S 9\}$,

rule 25) if the evaluation on Physics is good, and the evaluation on Literature is medium, and the evaluation on Philosophy is medium, then the student is rejected, $\quad\{S 14\}$.

These are all possible rules, however, there are several minimal subsets of decision rules. For example, one of them is constituted by the following nine rules: rule 1 ), rule 3 ), rule 9 ), rule 10 , rule 11 ), rule 15 ), rule 16 ), rule 17 ) and rule 21 ).

\section{Indiscernibility-based rough approximation of orthopairs}

Given the universe $U$, any pair $(X, Y)$, such that $X, Y \subseteq U, X \cap Y=\emptyset$, is an orthopair. ${ }^{9}$ Let us denote by $3^{U}$ the set of all the orthopairs, that is,

$$
3^{U}=\{(X, Y): X, Y \subseteq U, X \cap Y=\emptyset\} .
$$

Any orthopair $(X, Y)$ can be interpreted as representation of a concept $\mathcal{C}(X, Y)$ for which $X$ contains the elements from $U$ that for sure belong to $\mathcal{C}(X, Y)$ and $Y$ all the objects that for sure do not belong to $\mathcal{C}(X, Y)$. Observe that the information contained in an orthopair, that is the distinction between the positive part $X$, the negative part $Y$ and the uncertain part $U-X-Y$ of concept $\mathcal{C}(X, Y)$, can be represented also in terms of an interval set. In general, an interval set is a pair $(W, Z), W \subseteq Z \subseteq U$, where $W$ represents the positive part of a concept and $Z$ the possible part of a concept, that is the union of the positive and the doubtful part. Therefore, any orthopair $(X, Y)$ is equivalent to the interval set $(X, U-Y)$, while vice versa, any interval set $(W, Z)$ is equivalent to the orthopair $(W, U-Z)$. In more 
formal terms, considering the family $U^{\text {int }}$ of all the interval sets on the universe $U$, that is,

$$
U^{i n t}=\{(W, Z): W \subseteq Z \subseteq U\}
$$

we can consider the function $\mathcal{T}: 3^{U} \rightarrow U^{\text {int }}$ that assigns the corresponding interval set $(W, Z)$ to the orthopair $(X, Y)$, that is $\mathcal{T}(X, Y)=(X, U-Y)$. We shall consider also the inverse function of $\mathcal{T}$, that is, the function $\mathcal{T}^{-1}: U^{i n t} \rightarrow 3^{U}$ that to each interval set $(W, Z) \in U^{\text {int }}$ assigns the corresponding orthopair $(X, Y) \in 3^{U}$, such that $\mathcal{T}(X, Y)=(W, Z)$. We have $\mathcal{T}^{-1}(W, Z)=(W, Z-W)$.

Given any set of attributes $P \subseteq Q$, we can define the lower and the upper rough approximation of the orthopair $(X, Y)$, denoted by $\underline{I}_{P}^{\text {ortho }}(X, Y)$ and $\bar{I}_{P}^{\text {ortho }}(X, Y)$, respectively, as follows:

$$
\begin{aligned}
& \underline{I}_{P}^{\text {ortho }}(X, Y)=\left(\underline{I}_{P}(X), \underline{I}_{P}(Y)\right), \\
& \bar{I}_{P}^{\text {ortho }}(X, Y)=\left(\bar{I}_{P}(X), \bar{I}_{P}(Y)\right) .
\end{aligned}
$$

Observe that $\underline{I}_{P}^{\text {ortho }}(X, Y)$ is an orthopair. Indeed, $\underline{I}_{P}(X) \subseteq X$ as well as $\underline{I}_{P}(Y) \subseteq Y$ and, consequently, $X \cap Y=\emptyset$, imply $\underline{I}_{P}(X) \cap \underline{I}_{P}(Y)=\emptyset$. This is, however, not the case for $\bar{I}_{P}^{\text {ortho }}(X, Y)$ because, in general, $X \cap Y=\emptyset$ does not imply $\bar{I}_{P}(X) \cap$ $\bar{I}_{P}(Y)=\emptyset$. It is interesting to discuss the interpretation of orthopairs $\underline{I}_{P}^{\text {ortho }}(X, Y)$ and $\bar{I}_{P}^{\text {ortho }}(X, Y)$ with respect to the concept $\mathcal{C}(X, Y)$. For this it is important to distinguish between:

- vagueness, related to the uncertainty 'a priori', concerning the assignment of objects to the concept $\mathcal{C}(X, Y)$ at hand, and

- ambiguity, related to the granularity of knowledge, which requires that the 'a priori' assignment of objects is revised 'a posteriori' using rough approximations $\underline{I}_{P}^{\text {ortho }}(X, Y)$ and $\bar{I}_{P}^{\text {ortho }}(X, Y)$.

In this perspective, we can say that:

- $\underline{I}_{P}(X)$ is the set of objects from $U$ for which, on the basis of the available knowledge, there is no doubt that these objects for sure belong to the concept $\mathcal{C}(X, Y)$,

- $\underline{I}_{P}(Y)$ is the set of objects from $U$ for which, on the basis of the available knowledge there is no doubt that these objects for sure do not belong to the concept $\mathcal{C}(X, Y)$,

- $\bar{I}_{P}(X)$ is the set of objects from $U$ for which, on the basis of the available knowledge, there possibly is some doubt that these objects for sure belong to the concept $\mathcal{C}(X, Y)$,

- $\bar{I}_{P}(Y)$ is the set of objects from $U$ for which, on the basis of the available knowledge there possibly is some doubt that these objects for sure do not belong to the concept $\mathcal{C}(X, Y)$. 
The basic properties of rough set approximations are inherited by the rough approximations of orthopairs. To show them, let us consider the family $U^{(2)}$ of all pairs $(X, Y)$ of subsets in $U$, that is

$$
U^{(2)}=2^{U} \times 2^{U}=\{(X, Y): X \subseteq U, Y \subseteq U\} .
$$

Let us now introduce the following operations on $U^{(2)}$ :

for all $\left(X_{1}, Y_{1}\right),\left(X_{2}, Y_{2}\right) \in U^{(2)}$,

- union

$$
\left(X_{1}, Y_{1}\right) \cup^{(2)}\left(X_{2}, Y_{2}\right)=\left(X_{1} \cup X_{2}, Y_{1} \cup Y_{2}\right),
$$

- intersection

$$
\left(X_{1}, Y_{1}\right) \cap^{(2)}\left(X_{2}, Y_{2}\right)=\left(X_{1} \cap X_{2}, Y_{1} \cap Y_{2}\right),
$$

- difference

$$
\left(X_{1}, Y_{1}\right){ }^{(2)}\left(X_{2}, Y_{2}\right)=\left(X_{1}-X_{2}, Y_{1}-Y_{2}\right) .
$$

Let us also define the inclusion $\subseteq^{(2)}$ in $U^{(2)}$ as follows:

for all $\left(X_{1}, Y_{1}\right),\left(X_{2}, Y_{2}\right) \in U^{(2)}$,

$$
\left(X_{1}, Y_{1}\right) \subseteq^{(2)}\left(X_{2}, Y_{2}\right) \text { iff } X_{1} \subseteq X_{2} \text { and } Y_{1} \subseteq Y_{2} .
$$

Using the operations on $U^{(2)}$ defined above, we can present the properties of the lower and upper approximations $\underline{I}_{P}^{\text {ortho }}(X, Y)$ and $\bar{I}_{P}^{\text {ortho }}(X, Y)$ as follows.

For any $(X, Y),\left(X_{1}, Y_{1}\right),\left(X_{2}, Y_{2}\right) \in U^{(2)}$ and for any $P \subseteq Q$,

(1) $\underline{I}_{P}^{\text {ortho }}(X, Y) \subseteq^{(2)}(X, Y) \subseteq^{(2)} \bar{I}_{P}^{\text {ortho }}(X, Y)$,

(2) $\underline{I}_{P}^{\text {ortho }}(\emptyset, \emptyset)=\bar{I}_{P}^{\text {ortho }}(\emptyset, \emptyset)=(\emptyset, \emptyset)$,

(3) $\underline{I}_{P}(U, U)^{\text {ortho }}=\bar{I}_{P}^{\text {ortho }}(U, U)=(U, U)$,

(4) $\bar{I}_{P}^{\text {ortho }}\left[\left(X_{1}, Y_{1}\right) \cup{ }^{(2)}\left(X_{2}, Y_{2}\right)\right]=\bar{I}_{P}^{\text {ortho }}\left(X_{1}, Y_{1}\right) \cup^{(2)} \bar{I}_{P}^{\text {ortho }}\left(X_{2}, Y_{2}\right)$,

(5) $\underline{I}_{P}^{\text {ortho }}\left[\left(X_{1}, Y_{1}\right) \cap \cap^{(2)}\left(X_{2}, Y_{2}\right)\right]=\underline{I}_{P}^{\text {ortho }}\left(X_{1}, Y_{1}\right) \cap^{(2)} \underline{I}_{P}^{\text {ortho }}\left(X_{2}, Y_{2}\right)$,

(6) $\left(X_{1}, Y_{1}\right) \subseteq{ }^{(2)}\left(X_{2}, Y_{2}\right) \Rightarrow \underline{I}_{P}^{\text {ortho }}\left(X_{1}, Y_{1}\right) \subseteq^{(2)} \underline{I}_{P}^{\text {ortho }}\left(X_{2}, Y_{2}\right)$,

(7) $\left(X_{1}, Y_{1}\right) \subseteq{ }^{(2)}\left(X_{2}, Y_{2}\right) \Rightarrow \bar{I}_{P}^{\text {ortho }}\left(X_{1}, Y_{1}\right) \subseteq \subseteq^{(2)} \bar{I}_{P}^{\text {ortho }}\left(X_{2}, Y_{2}\right)$,

(8) $\underline{I}_{P}^{\text {ortho }}\left[\left(X_{1}, Y_{1}\right) \cup(2)\left(X_{2}, Y_{2}\right)\right] \underline{ }^{(2)} \underline{I}_{P}^{\text {ortho }}\left(X_{1}, Y_{1}\right) \cup \cup^{(2)} \underline{I}_{P}^{\text {ortho }}\left(X_{2}, Y_{2}\right)$,

(9) $\bar{I}_{P}^{\text {ortho }}\left[\left(X_{1}, Y_{1}\right) \cap^{(2)}\left(X_{2}, Y_{2}\right)\right] \subseteq^{(2)} \bar{I}_{P}^{\text {ortho }}\left(X_{1}, Y_{1}\right) \cap^{(2)} \bar{I}_{P}^{\text {ortho }}\left(X_{2}, Y_{2}\right)$,

(10) $\underline{I}_{P}^{\text {ortho }}\left[(U, U)-{ }^{(2)}(X, Y)\right]=(U, U)-{ }^{(2)} \bar{I}_{P}^{\text {ortho }}(X, Y)$,

(11) $\bar{I}_{P}^{\text {ortho }}\left[(U, U)-{ }^{(2)}(X, Y)\right]=(U, U)-{ }^{(2)} \underline{I}_{P}^{\text {ortho }}(X, Y)$,

(12) $\underline{I}_{P}^{\text {ortho }}\left[\underline{I}_{P}^{\text {ortho }}(X, Y)\right]=\bar{I}_{P}^{\text {ortho }}\left[\underline{I}_{P}^{\text {ortho }}(X, Y)\right]=\underline{I}_{P}^{\text {ortho }}(X, Y)$,

(13) $\bar{I}_{P}^{\text {ortho }}\left[\bar{I}_{P}^{\text {ortho }}(X, Y)\right]=\underline{I}_{P}^{\text {ortho }}\left[\bar{I}_{P}^{\text {ortho }}(X, Y)\right]=\bar{I}_{P}^{\text {ortho }}(X, Y)$.

With respect to property $(10)$, observe that $(U, U){ }^{(2)}(X, Y)$ is not an orthopair because

$$
(U, U)-^{(2)}(X, Y)=(U-X, U-Y)
$$


and, unless $X \cup Y=U$,

$$
(U-X) \cap(U-Y)=U-(X \cup Y) \neq \emptyset .
$$

Therefore, there is a small abuse of notation because the operators $\underline{I}_{P}^{\text {ortho }}$ and $\bar{I}_{P}^{\text {ortho }}$ are applied to the pair $(U, U)-{ }^{(2)}(X, Y)=(U-X, U-Y)$ which is not an orthopair. Nevertheless, accepting this abuse of notation, property (10) holds in the sense that

$$
\begin{gathered}
\underline{I}_{P}^{\text {ortho }}\left[(U, U)-{ }^{(2)}(X, Y)\right]=\underline{I}_{P}^{\text {ortho }}[(U-X, U-Y)]= \\
\left(\underline{I}_{P}(U-X), \underline{I}_{P}(U-Y)\right)=\left(U-\bar{I}_{P}(X), U-\bar{I}_{P}(Y)\right)=(U, U)-{ }^{(2)} \bar{I}_{P}^{\text {ortho }}(X, Y) .
\end{gathered}
$$

Clearly, an analogous caveat holds for property (11). Observe that there is an abuse of notation of the same type in property (13) where operators $\underline{I}_{P}^{\text {ortho }}$ and $\bar{I}_{P}^{\text {ortho }}$ are applied to $\bar{I}_{P}^{\text {ortho }}(X, Y)$ which, as already observed, is not always an orthopair.

Similarly to the orthopairs, for any interval set $(W, Z)$, we can define the lower rough approximation $\underline{I}_{P}^{\text {int }}(W, Z)$ and the upper rough approximation $\bar{I}_{P}^{\text {int }}(W, Z)$, as follows:

$$
\begin{aligned}
& \underline{I}_{P}^{i n t}(W, Z)=\left(\underline{I}_{P}(W), \bar{I}_{P}(Z)\right), \\
& \bar{I}_{P}^{i n t}(W, Z)=\left(\bar{I}_{P}(W), \underline{I}_{P}(Z)\right) .
\end{aligned}
$$

Observe that the definition of the rough approximation of interval sets is coherent with the rough approximation of orthopairs, because for any $(W, Z) \in U^{i n t}$,

$$
\begin{aligned}
& \underline{I}_{P}^{\text {int }}(W, Z)=\mathcal{T}\left(\underline{I}_{P}^{\text {ortho }}\left(\mathcal{T}^{-1}(W, Z)\right)\right), \\
& \bar{I}_{P}^{\text {int }}(W, Z)=\mathcal{T}\left(\bar{I}_{P}^{\text {ortho }}\left(\mathcal{T}^{-1}(W, Z)\right)\right) .
\end{aligned}
$$

This means that we can obtain the rough approximation $\underline{I}_{P}^{\text {int }}(W, Z)$ and $\bar{I}_{P}^{i n t}(W, Z)$ of the interval set $(W, Z) \in U^{\text {int }}$, as transformation through $\mathcal{T}$ of the rough approximation $\underline{I}_{P}^{\text {ortho }}\left(\mathcal{T}^{-1}(W, Z)\right)$ and $\bar{I}_{P}^{\text {ortho }}\left(\mathcal{T}^{-1}(W, Z)\right)$ of the orthopair $\mathcal{T}^{-1}(W, Z)$ corresponding to the interval set $(W, Z)$.

We can also define binary operations $\cup^{\text {int }}$ and $\cap^{\text {int }}$ on $U^{(2)}$ permitting to represent properties of rough approximations of interval sets corresponding to the anologous operations $\cup^{(2)}$ and $\cap^{(2)}$ introduced above for the orthopairs, that is, for $\left(W_{1}, Z_{1}\right),\left(W_{2}, Z_{2}\right) \in U^{(2)}$ we have:

- union

$$
\left(W_{1}, Z_{1}\right) \cup^{\text {int }}\left(W_{2}, Z_{2}\right)=\mathcal{T}\left(\mathcal{T}^{-1}\left(W_{1}, Z_{1}\right) \cup^{\text {ortho }} \mathcal{T}^{-1}\left(W_{2}, Z_{2}\right)\right)=\left(W_{1} \cup W_{2}, Z_{1} \cap Z_{2}\right),
$$

- intersection

$$
\left(W_{1}, Z_{1}\right) \cap^{\text {int }}\left(W_{2}, Z_{2}\right)=\mathcal{T}\left(\mathcal{T}^{-1}\left(W_{1}, Z_{1}\right) \cap^{\text {ortho }} \mathcal{T}^{-1}\left(W_{2}, Z_{2}\right)\right)=\left(W_{1} \cap W_{2}, Z_{1} \cup Z_{2}\right) .
$$


Moreover, the inclusion $\subseteq^{\text {int }}$ on $U^{(2)}$, useful to present properties of rough approximations of interval sets can be defined as follows: for all $\left(W_{1}, Z_{1}\right),\left(W_{2}, Z_{2}\right) \subseteq U^{(2)}$,

$$
\begin{gathered}
\left(W_{1}, Z_{1}\right) \subseteq^{\text {int }}\left(W_{2}, Z_{2}\right) \\
\text { iff } \\
\mathcal{T}^{-1}\left(W_{1}, Z_{1}\right) \subseteq^{(2)} \mathcal{T}^{-1}\left(W_{2}, Z_{2}\right) \\
\text { iff } \\
W_{1} \subseteq W_{2} \text { and } Z_{1} \supseteq Z_{2} .
\end{gathered}
$$

Using the operations $\cup^{i n t}, \cap^{i n t}$ and $-^{(2)}$, and the inclusion $\subseteq^{\text {int }}$, we can present the properties of the lower and upper approximations $\underline{I}_{P}^{\text {ortho }}(X, Y)$ and $\bar{I}_{P}^{\text {ortho }}(X, Y)$ as follows.

For any $(W, Z) \in U^{\text {int }}$ and for any $P \subseteq Q$,

(1) $\underline{I}_{P}^{i n t}(W, Z) \subseteq^{\text {int }}(W, Z) \subseteq \subseteq^{\text {int }} \bar{I}_{P}^{i n t}(W, Z)$,

(2) $\underline{I}_{P}^{\text {int }}(\emptyset, \emptyset)=\bar{I}_{P}^{\text {int }}(\emptyset, \emptyset)=(\emptyset, \emptyset)$,

(3) $\underline{I}_{P}^{\text {int }}(U, U)=\bar{I}_{P}^{\text {int }}(U, U)=(U, U)$,

(4) $\bar{I}_{P}^{i n t}\left[\left(W_{1}, Z_{1}\right) \cup\right.$ int $\left.\left(W_{2}, Z_{2}\right)\right]=\bar{I}_{P}^{i n t}\left(W_{1}, Z_{1}\right) \cup$ int $\bar{I}_{P}^{\text {int }}\left(W_{2}, Z_{2}\right)$,

(5) $\underline{I}_{P}^{\text {int }}\left[\left(W_{1}, Z_{1}\right) \cap \cap^{\text {int }}\left(W_{2}, Z_{2}\right)\right]=\underline{I}_{P}^{\text {int }}\left(W_{1}, Z_{1}\right) \cap^{\text {int }} \underline{I}_{P}^{\text {int }}\left(W_{2}, Z_{2}\right)$,

(6) $\left(W_{1}, Z_{1}\right) \subseteq \subseteq^{\text {int }}\left(W_{2}, Z_{2}\right) \Rightarrow \underline{I}_{P}^{\text {int }}\left(W_{1}, Z_{1}\right) \subseteq^{\text {int }} \underline{I}_{P}^{\text {int }}\left(W_{2}, Z_{2}\right)$,

(7) $\left(W_{1}, Z_{1}\right)^{\text {int }} \subseteq^{\text {int }}\left(W_{2}, Z_{2}\right) \Rightarrow \bar{I}_{P}^{i n t}\left(W_{1}, Z_{1}\right) \subseteq^{\text {int }} \bar{I}_{P}^{\text {int }}\left(W_{2}, Z_{2}\right)$,

(8) $\underline{I}_{P}^{\text {int }}\left[\left(W_{1}, Z_{1}\right) \cup\right.$ int $\left.\left(W_{2}, Z_{2}\right)\right] \supseteq^{\text {int }} \underline{I}_{P}^{\text {int }}\left(W_{1}, Z_{1}\right) \cup \cup^{\text {int }} \underline{I}_{P}^{\text {int }}\left(W_{2}, Z_{2}\right)$,

(9) $\bar{I}_{P}^{\text {int }}\left[\left(W_{1}, Z_{1}\right) \cap\right.$ int $\left.\left(W_{2}, Z_{2}\right)\right] \subseteq \subseteq^{\text {int }} \bar{I}_{P}^{\text {int }}\left(W_{1}, Z_{1}\right) \cap \cap^{\text {int }} \bar{I}_{P}^{\text {int }}\left(W_{2}, Z_{2}\right)$,

(10) $\underline{I}_{P}^{\text {int }}\left[(U, U)-{ }^{(2)}(W, Z)\right]=(U, U)-{ }^{(2)} \bar{I}_{P}^{i n t}(W, Z)$,

(11) $\bar{I}_{P}^{\text {int }}\left[(U, U)-{ }^{(2)}(W, Z)\right]=(U, U)-{ }^{(2)} \underline{I}_{P}^{\text {int }}(W, Z)$,

(12) $\underline{I}_{P}^{\text {int }}\left[\underline{I}_{P}(W, Z)\right]=\bar{I}_{P}\left[\underline{I}_{P}^{\text {int }}(W, Z)\right]=\underline{I}_{P}^{\text {int }}(W, Z)$,

(13) $\bar{I}_{P}^{i n t}\left[\bar{I}_{P}^{i n t}(W, Z)\right]=\underline{I}_{P}^{i n t}\left[\bar{I}_{P}^{i n t}(W, Z)\right]=\bar{I}_{P}^{i n t}(W, Z)$.

Observe that analogous remarks related to abuse of notation provided for properties (10), (11) and (13) of rough approximation operators $\underline{I}_{P}^{\text {ortho }}$ and $\bar{I}_{P}^{\text {ortho }}$ on orthopairs hold for properties (10), (11) and (13) of rough approximation operators $\underline{I}_{P}^{\text {int }}$ and $\bar{I}_{P}^{\text {int }}$ on interval sets.

Generalizing the analogous definition given for the classical Indiscernibilitybased Rough Set Approach (IRSA), taking $P \subseteq Q$ and $p \in P$, it is said that attribute $p$ is superfluous in $P$ with respect to approximation of the orthopair $(X, Y) \in 3^{U}$ if $\underline{I}_{P}(X, Y)=\underline{I}_{P-\{p\}}(X, Y)$; otherwise, $p$ is indispensable in $P$. Also for the rough approximation of orthopairs, the subset of $Q$ containing all the indispensable attributes constitutes the core, as well as any minimal (with respect to 
inclusion) subset $P \subseteq Q$, such that $\underline{P}(X, Y)=\underline{Q}(X, Y)$, is called a reduct of $Q$ with respect to the approximation of $(X, Y)$.

Taking into account the vague concept $(X, Y) \in 3^{U}$, one can induce the following decision rules:

- certain decision rules, providing profile descriptions for objects belonging to $\underline{I}_{Q}(X)$

- certain decision rules, providing profile descriptions for objects belonging to $\underline{I}_{Q}(Y)$,

- certain decision rules, providing profile descriptions for objects belonging to $\underline{I}_{Q}(U-Y)$,

- certain decision rules, providing profile descriptions for objects belonging to $\underline{I}_{Q}(U-X)$

- possible decision rules, providing profile descriptions for objects belonging to $\bar{I}_{Q}(X)$

- possible decision rules, providing profile descriptions for objects belonging to $\bar{I}_{Q}(Y)$

- possible decision rules, providing profile descriptions for objects belonging to $\bar{I}_{Q}(U-Y)$,

- possible decision rules, providing profile descriptions for objects belonging to $\bar{I}_{Q}(U-X)$.

\subsection{Didactic example}

Continuing the example introduced in subsection 2.1, after looking at results supplied by the rough approximation based on indiscernibility, the committee decided to distinguish more finely between students that should be accepted for sure ("accepted"), students that should be rejected for sure ("rejected") and other students for whom the decision is doubtful ("?"), as shown in Table 2.

Observe that student $S 6$ and student $S 9$ constitute a case of ambiguity: indeed they are indiscernible (because they have the same evaluations on all subjects), but for $S 6$ the acceptance is doubtful while for $S 9$ there is a sure rejection. Notice also that they became ambiguous when the committee passed from Table 1, distinguishing between acceptance and rejection only, to the finer information of Table 2 which distinguishes between sure acceptance, sure rejection and doubtful situations.

There is only one reduct that is also the core:

$$
\text { Red }_{\text {ortho }}=\{\text { Mathematics, Physics, Philosophy }\} .
$$

The following certain decision rules can been induced (between braces there are id's of students supporting the corresponding rule):

rule 1') if the evaluation on Mathematics is good, and the evaluation on Physics is good, then the student is accepted, $\{S 1, S 2\}$, 
Table 2. Exemplary decisions on admission of candidate students

\begin{tabular}{|c|c|c|c|c|c|}
\hline Student & Mathematics & Physics & Literature & Philosophy & Admission/rejection \\
\hline$S 1$ & good & good & bad & good & accepted \\
\hline$S 2$ & good & good & good & medium & accepted \\
\hline$S 3$ & medium & good & medium & good & $?$ \\
\hline$S 4$ & medium & medium & medium & bad & $?$ \\
\hline$S 5$ & bad & medium & medium & good & rejected \\
\hline$S 6$ & medium & medium & medium & good & $?$ \\
\hline$S 7$ & bad & medium & good & good & rejected \\
\hline$S 8$ & good & medium & bad & good & $?$ \\
\hline$S 9$ & medium & medium & medium & good & rejeced \\
\hline$S 10$ & medium & medium & medium & medium & $?$ \\
\hline$S 11$ & bad & medium & good & medium & rejected \\
\hline$S 12$ & medium & good & bad & bad & $?$ \\
\hline$S 13$ & good & bad & medium & medium & rejected \\
\hline$S 14$ & bad & good & medium & medium & rejected \\
\hline$S 15$ & bad & bad & good & medium & rejected \\
\hline
\end{tabular}

rule 2') if the evaluation on Mathematics is good, and the evaluation on Literature is good, then the student is accepted, $\{S 2\}$,

rule 3') if the evaluation on Physics is good, and the evaluation on Literature is good, then the student is accepted, $\{S 2\}$,

rule 4') if the evaluation on Physics is good, and the evaluation on Literature is bad, and the evaluation on Philosophy is good, then the student is accepted, $\{S 1\}$,

rule 5') if the evaluation on Mathematics is medium, and the evaluation on Physics is good, then the acceptance of the student is doubtful, $\{S 3, S 12\}$,

rule 6') if the evaluation on Mathematics is good, and the evaluation on Physics is medium, then the acceptance of the student is doubtful, $\{S 8\}$,

rule $7^{\prime}$ ) if the evaluation on Mathematics is medium, and the evaluation on Literature is bad, then the acceptance of the student is doubtful, $\{S 12\}$,

rule $8^{\prime}$ ) if the evaluation on Physics is medium, and the evaluation on Literature is bad, then the acceptance of the student is doubtful, $\{S 8\}$,

rule 9') if the evaluation on Philosophy is bad, then the acceptance of the student is doubtful, $\{S 4, S 12\}$,

rule 10') if the evaluation on Mathematics is medium, and the evaluation on Philosophy is bad, then the acceptance of the student is doubtful, $\{S 4, S 12\}$, 
rule 11') if the evaluation on Physics is good, and the evaluation on Literature is medium, and the evaluation on Philosophy is good, then the acceptance of the student is doubtful, $\{S 3\}$,

rule 12') if the evaluation on Physics is medium, and the evaluation on Literature is medium, and the evaluation on Philosophy is medium, then the acceptance of the student is doubtful, $\{S 10\}$,

rule 13') if the evaluation on Mathematics is bad, then the student is rejected, $\{S 5, S 7, S 11, S 14, S 15\}$,

rule 14') if the evaluation on Physics is bad, then the student is rejected, $\{S 13, S 15\}$,

rule 15') if the evaluation on Mathematics is good, and the evaluation on Literature is medium, then the student is rejected, $\{S 13\}$,

rule 16') if the evaluation on Physics is medium, and the evaluation on Literature is good, then the student is rejected, $\{S 7, S 11\}$,

rule 17') if the evaluation on Literature is good, and the evaluation on Philosophy is good, then the student is rejected, $\{S 7\}$,

rule 18') if the evaluation on Physics is good, and the evaluation on Literature is medium, and the evaluation on Philosophy is medium, then the student is rejected, $\{S 14\}$,

There are several minimal sets of decision rules. One of them is composed by the following decision rules: rule1'), rule5'), rule 9'), rule 10'), rule 13'), rule 14').

\section{Dominance-based Rough Set Approach}

In this section, we recall the Dominance-based Rough Set Approach (DRSA). ${ }^{15}$ Let us consider an information table $\mathcal{I} \mathcal{T}=\langle U, Q, V, v\rangle$. Without loss of generality suppose that $V_{q} \subseteq \Re$ such that $v(x, q) \in \Re$ for each $q=1, \ldots, n$, and, for all objects $x, y \in U, v(x, q) \geq v(y, q)$ means that " $x$ is at least as good as $y$ with respect to attribute (criterion) $q$ ", which is denoted by $x \succeq_{q} y$. Therefore, we suppose that $\succeq_{q}$ is a complete preorder, i.e., a strongly complete and transitive binary relation, defined on $U$ on the basis of evaluations $v(\cdot, q)$.

The key idea of DRSA is upward and downward approximation of sets of objects by granules of knowledge generated by criteria. These granules are dominance cones in the space of criteria values.

We say that $x$ dominates $y$ with respect to set of criteria $P \subseteq Q$ (shortly, $x$ $P$-dominates $y$ ), denoted by $x D_{P} y$, if for every criterion $q \in P, v(x, q) \geq v(y, q)$. The relation of $P$-dominance is reflexive and transitive, i.e., it is a partial preorder.

Given a set of criteria $P \subseteq Q$ and $x \in U$, the granules of knowledge used for approximation in DRSA are: 
- a set of objects dominating $x$, called P-dominating set, $D_{P}^{+}(x)=\left\{y \in U: y D_{P} x\right\}$,

- a set of objects dominated by $x$, called $P$-dominated set, $D_{P}^{-}(x)=\left\{y \in U: x D_{P} y\right\}$.

Given $P \subseteq Q$ and $X \subseteq U$, the $P$-upward-lower and the $P$-upward-upper approximation of $X$ are defined in terms of unions of $P$-dominating sets $D_{P}^{+}(x)$, as follows:

$$
\begin{gathered}
\underline{D}_{P}^{+}(X)=\bigcup_{x \in U: D_{P}^{+}(x) \subseteq X} D_{P}^{+}(x), \\
\bar{D}_{P}^{+}(X)=\bigcup_{x \in U: D_{P}^{-}(x) \cap X \neq \emptyset} D_{P}^{+}(x) .
\end{gathered}
$$

Analogously, the $P$-downward-lower and the $P$-downward-upper approximation of $X$ are defined in terms of unions of $P$-dominated sets $D_{P}^{-}(x)$, as follows:

$$
\begin{gathered}
\underline{D}_{P}^{-}(X)=\bigcup_{x \in U: D_{P}^{-}(x) \subseteq X} D_{P}^{-}(x), \\
\bar{D}_{P}^{-}(X)=\bigcup_{x \in U: D_{P}^{+}(x) \cap X \neq \emptyset} D_{P}^{-}(x) .
\end{gathered}
$$

The rough approximations $\underline{D}_{P}^{+}(X), \bar{D}_{P}^{+}(X), \underline{D}_{P}^{-}(X)$ and $\bar{D}_{P}^{-}(X)$ can also be formulated as follows:

$$
\begin{gathered}
\underline{D}_{P}^{+}(X)=\left\{x \in U: D_{P}^{+}(x) \subseteq X\right\}, \\
\bar{D}_{P}^{+}(X)=\left\{x \in U: D_{P}^{-}(x) \cap X \neq \emptyset\right\}, \\
\underline{D}_{P}^{-}(X)=\left\{x \in U: D_{P}^{-}(x) \subseteq X\right\}, \\
\bar{D}_{P}^{-}(X)=\left\{x \in U: D_{P}^{+}(x) \cap X \neq \emptyset\right\} .
\end{gathered}
$$

The lower and upper approximations $\underline{D}_{P}^{+}(X), \bar{D}_{P}^{+}(X), \underline{D}_{P}^{-}(X)$ and $\bar{D}_{P}^{-}(X)$ satisfy the following properties.

Theorem 2.15,22 For any $X, Y \subseteq U$ and for any $P \subseteq Q$,

(1) $\underline{D}_{P}^{+}(X) \subseteq X \subseteq \bar{D}_{P}^{+}(X), \underline{D}_{P}^{-}(X) \subseteq X \subseteq \bar{D}_{P}^{-}(X)$,

(2) $\underline{D}_{P}^{+}(\emptyset)=\bar{D}_{P}^{+}(\emptyset)=\emptyset, \underline{D}_{P}^{-}(\emptyset)=\bar{D}_{P}^{-}(\emptyset)=\emptyset$,

(3) $\underline{D}_{P}^{+}(U)=\bar{D}_{P}^{+}(U)=U, \underline{D}_{P}^{-}(U)=\bar{D}_{P}^{-}(U)=U$,

(4) $\bar{D}_{P}^{+}(X \cup Y)=\bar{D}_{P}^{+}(X) \cup \bar{D}_{P}^{+}(Y), \bar{D}_{P}^{-}(X \cup Y)=\bar{D}_{P}^{-}(X) \cup \bar{D}_{P}^{-}(Y)$,

(5) $\underline{D}_{P}^{+}(X \cap Y)=\underline{D}_{P}^{+}(X) \cap \underline{D}_{P}^{+}(Y), \underline{D}_{P}^{-}(X \cap Y)=\underline{D}_{P}^{-}(X) \cap \underline{D}_{P}^{-}(Y)$, 
(6) $X \subseteq Y \Rightarrow \underline{D}_{P}^{+}(X) \subseteq \underline{D}_{P}^{+}(Y)$ and $\underline{D}_{P}^{-}(X) \subseteq \underline{D}_{P}^{-}(Y)$,

(7) $X \subseteq Y \Rightarrow \bar{D}_{P}(X) \subseteq \bar{D}_{P}(Y)$ and $\bar{D}_{P}^{-}(X) \subseteq \bar{D}_{P}^{-}(Y)$,

(8) $\underline{D}_{P}^{+}(X \cup Y) \supseteq \underline{D}_{P}^{+}(X) \cup \underline{D}_{P}^{+}(Y), \underline{D}_{P}^{-}(X \cup Y) \supseteq \underline{D}_{P}^{-}(X) \cup \underline{D}_{P}^{-}(Y)$,

(9) $\bar{D}_{P}^{+}(X \cap Y) \subseteq \bar{D}_{P}^{+}(X) \cap \bar{D}_{P}^{+}(Y), \bar{D}_{P}^{-}(X \cap Y) \subseteq \bar{D}_{P}^{-}(X) \cap \bar{D}_{P}^{-}(Y)$,

(10) $\underline{D}_{P}^{+}(U-X)=U-\bar{D}_{P}^{-}(X), \underline{D}_{P}^{-}(U-X)=U-\bar{D}_{P}^{+}(X)$,

(11) $\bar{D}_{P}^{+}(U-X)=U-\underline{D}_{P}^{-}(X), \bar{D}_{P}^{-}(U-X)=U-\underline{D}_{P}^{+}(X)$,

(12) $\underline{D}_{P}^{+}\left[\underline{D}_{P}^{+}(X)\right]=\bar{D}_{P}^{+}\left[\underline{D}_{P}^{+}(X)\right]=\underline{D}_{P}^{+}(X)$, $\underline{D}_{P}^{-}\left[\underline{D}_{P}^{-}(X)\right]=\bar{D}_{P}^{-}\left[\underline{D}_{P}^{-}(X)\right]=\underline{D}_{P}^{-}(X)$,

(13) $\bar{D}_{P}^{+}\left[\bar{D}_{P}^{+}(X)\right]=\underline{D}_{P}^{+}\left[\bar{D}_{P}^{+}(X)\right]=\bar{D}_{P}^{+}(Y)$, $\bar{D}_{P}^{-}\left[\bar{D}_{P}^{-}(X)\right]=\underline{D}_{P}^{-}\left[\bar{D}_{P}^{-}(X)\right]=\bar{D}_{P}^{-}(Y)$.

In the context of DRSA, the reduct has a definition analogous to that one in the context of IRSA. More precisely, given $P \subseteq Q$ and $p \in P$, attribute $p$ is

- superfluous in $P$ with respect to upward approximation of $X \subseteq U$ if $\underline{D}_{P}^{+}(X)=$ $\underline{D}_{P-\{p\}}^{+}(X)$, which is equivalent to $\underline{D}_{P}^{-}(U-X)=\underline{D}_{P-\{p\}}^{-}(U-X)$; otherwise, $p$ is upward indispensable in $P$;

- superfluous in $P$ with respect to downward approximation of $X \subseteq U$ if $\underline{D}_{P}^{-}(X)=\underline{D}_{P-\{p\}}^{-}(X)$, which is equivalent to $\underline{D}_{P}^{+}(U-X)=\underline{D}_{P-\{p\}}^{+}(U-X)$; otherwise, $p$ is downward indispensable in $P$.

The subset of $Q$ containing all the upward indispensable attributes with respect to $X$ is known as the upward core. Analogously, the set composed fo all the downward indispensable attributes with respect to $X$ is the downward core. Any minimal (with respect to inclusion) subset $P \subseteq Q$, such that $\underline{D}_{P}^{+}(X)=\underline{D}_{Q}^{+}(X)$, is called an upward reduct with respect to the approximation of $X$. In the same way, a minimal subset $P \subseteq Q$, such that $\underline{D}_{P}^{-}(X)=\underline{D}_{Q}^{-}(X)$, is called a downward reduct with respect to the approximation of $X$.

The dominance-based rough approximations $\underline{D}_{P}^{+}(X), \underline{D}_{P}^{-}(X), \bar{D}_{P}^{+}(X)$ and $\bar{D}_{P}^{-}(X)$ can serve to induce a generalized description of objects related to the set $X$ in terms of "if ..., then ..." decision rules (see, e.g., ${ }^{23}$ ). If $X \subseteq U$ is a concept positively correlated with the evaluations supplied by the attribute in the information table, that is, the larger $v(x, q), q \in Q$, the more credible the membership of $x \in U$ to $X$, the decision rules induced under a hypothesis that objects belonging to $\underline{D}_{P}^{+}(X)$ are positive examples, and all the others are negative, suggest a certain assignment to $X$. Instead, if $X \subseteq U$ is a concept negatively correlated with the evaluations supplied by the attribute in the information table, that is, the smaller $v(x, q)$ the more credible the membership to $X$, the decision rules suggesting a certain assignment to $X$ have to be induced under a hypothesis that objects belonging to $\underline{D}_{P}^{-}(X)$ are positive examples, and all the others are negative. On the other hand, the decision rules induced under a hypothesis that objects belonging to $\bar{D}_{P}^{+}(X)$, in case $X \subseteq U$ is positively correlated with the evaluations $v(x, q), q \in Q$, or $\bar{D}_{P}^{-}(X)$, in case $X \subseteq U$ is negatively correlated with the evaluations $v(x, q), q \in Q$, are pos- 
itive examples, and all the others are negative, suggest a possible assignment to $X$. Therefore, the following four types of decision rules can be considered:

1) certain $\mathrm{D}_{\geq-}$decision rules, providing lower profile descriptions for objects belonging to $\underline{D}_{P}^{+}(X)$, in case membership to $X$ is positively correlated with evaluations $v(x, q), q \in Q$ :

if $v\left(x, q_{1}\right) \geq r_{q_{1}}$ and $\ldots$ and $v\left(x, q_{p}\right) \geq r_{q_{p}}$, then $x \in X$, $\left\{q_{1}, \ldots, q_{p}\right\} \subseteq Q, r_{q_{1}}, \ldots, r_{q_{p}} \in \Re$

2) possible $\mathrm{D}_{\geq-}$-decision rules, providing lower profile descriptions for objects belonging to $\bar{D}_{P}^{+}(X)$ :

if $v\left(x, q_{1}\right) \geq r_{q_{1}}$ and $\ldots$ and $v\left(x, q_{p}\right) \geq r_{q_{p}}$, then $x$ possibly belongs to $X$, $\left\{q_{1}, \ldots, q_{p}\right\} \subseteq Q, r_{q_{1}}, \ldots, r_{q_{p}} \in \Re$

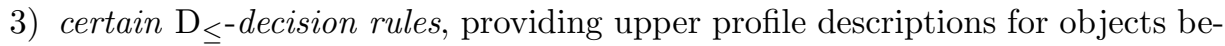
longing to $\underline{D}_{P}^{-}(X)$ :

if $v\left(x, q_{1}\right) \leq r_{q_{1}}$ and $\ldots$ and $v\left(x, q_{1}\right) \leq r_{q_{p}}$, then $x \in X$, $\left\{q_{1}, \ldots, q_{p}\right\} \subseteq Q, r_{q_{1}}, \ldots, r_{q_{p}} \in \Re$;

4) possible $\mathrm{D}_{\leq}$-decision rules, providing upper profile descriptions for objects belonging to $\bar{D}_{P}^{-}(X)$ :

if $v\left(x, q_{1}\right) \leq r_{q_{1}}$ and $\ldots$ and $v\left(x, q_{p}\right) \leq r_{q_{p}}$, then $x$ possibly belongs to $X$, $\left\{q_{1}, \ldots, q_{p}\right\} \subseteq Q, r_{q_{1}}, \ldots, r_{q_{p}} \in \Re$.

\subsection{Didactic example}

Let us consider again the example introduced in subsection 2.1 and continued in subsection 3.1. After looking at results supplied by rough approximations based on indiscernibility, both for the precise (acceptance/rejection) and the imprecise (acceptance/rejection/?) assignments shown in Table 1 and Table 2, respectively, the committee decided to consider the ordinal nature of the data related to acceptance and rejection of candidate students. Indeed, an evaluation "good" is not only different from an evaluation "medium", because, clearly, "good" is better than "medium". In the same way, "medium" is better than "bad". Moreover, a candidate "accepted" has an overall evaluation better than a candidate "rejected". Therefore, DRSA was applied on Table 1 and a single reduct that is also the core was discovered. It is composed of all the four subjects. The following certain decision rules were induced:

rule 1") if the evaluation on Mathematics is (at least) good, and the evaluation on Physics is at least medium, then the student is accepted, $\{S 1, S 2, S 8\}$,

rule 2") if the evaluation on Mathematics is (at least) good, and the evaluation on Literature is (at least) good, then the student is accepted, $\{S 2\}$,

rule 3") if the evaluation on Physics is (at least) good, and the evaluation on Literature is (at least) good, then the student is accepted, $\{S 2\}$, 
rule 4") if the evaluation on Mathematics is at least medium, and the evaluation on Physics is (at least) good, and the evaluation on Literature is (at least) good, then the student is accepted, $\{S 2, S 3\}$,

rule 5") if the evaluation on Mathematics is (at least) good, and the evaluation on Philosophy is (at least) good, then the student is accepted, $\{S 1, S 8\}$,

rule 6") if the evaluation on Physics is (at least) good, and the evaluation on Philosophy is (at least) good, then the student is accepted, $\{S 1, S 3\}$,

rule 7") if the evaluation on Mathematics is (at most) bad, then the student is rejected, $\quad\{S 5, S 7, S 11, S 14, S 15\}$,

rule 8") if the evaluation on Physics is (at most) bad, then the student is rejected, $\{S 13, S 15\}$,

rule 9") if the evaluation on Mathematics is at most medium, and the evaluation on Literature is (at most) bad, then the student is rejected, $\{S 12\}$,

rule 10") if the evaluation on Literature is (at most) bad, and the evaluation on Philosophy is (at most) bad, then the student is rejected, $\{S 12\}$.

\section{Dominance-based rough approximations of orthopairs and interval sets}

Given any set of attributes $P \subset Q$, we can define the lower and the upper dominance-based rough approximations of the orthopair $(X, Y) \in U^{(2)}$ as follows:

$$
\begin{gathered}
\underline{D}_{P}^{+}(X, Y)=\left(\underline{D}_{P}^{+}(X), \underline{D}_{P}^{-}(Y)\right), \\
\underline{D}_{P}^{-}(X, Y)=\left(\underline{D}_{P}^{-}(X), \underline{D}_{P}^{+}(Y)\right), \\
\bar{D}_{P}^{+}(X, Y)=\left(\bar{D}_{P}^{+}(X), \bar{D}_{P}^{-}(Y)\right), \\
\bar{D}_{P}^{-}(X, Y)=\left(\bar{D}_{P}^{-}(X), \bar{D}_{P}^{+}(Y)\right) .
\end{gathered}
$$

Also in case of dominance-based rough approximations, $\underline{D}_{P}^{+}(X, Y)$ and $\underline{D}_{P}^{-}(X, Y)$ are orthopairs, while, in general, this is not the case for $\bar{D}_{P}^{+}(X, Y)$ and $\bar{D}_{P}^{-}(X, Y)$.

By means of operations $\cup^{(2)}, \cap^{(2)},-^{(2)}$ and the inclusion $\subseteq{ }^{(2)}$, we can express the properties of the dominance-based rough approximations of orthopairs as follows.

For any $(X, Y),\left(X_{1}, Y_{1}\right),\left(X_{2}, Y_{2}\right) \in 3^{U}$ and for any $P \subseteq Q$,

(1) $\underline{D}_{P}^{+}(X, Y) \subseteq \underline{c}^{(2)}(X, Y) \subseteq \underline{\subseteq}^{(2)} \bar{D}_{P}^{+}(X, Y)$, $\underline{D}_{P}^{-}(X, Y) \subseteq \subseteq^{(2)}(X, Y) \subseteq \subseteq^{(2)} \bar{D}_{P}^{-}(X, Y)$,

(2) $\underline{D}_{P}^{+}(\emptyset, \emptyset)=\bar{D}_{P}^{+}(\emptyset, \emptyset)=(\emptyset, \emptyset), \underline{D}_{P}^{-}(\emptyset, \emptyset)=\bar{D}_{P}^{-}(\emptyset, \emptyset)=(\emptyset, \emptyset)$,

(3) $\underline{D}_{P}^{+}(U, U)=\bar{D}_{P}^{+}(U, U)=(U, U), \underline{D}_{P}^{-}(U, U)=\bar{D}_{P}^{-}(U, U)=(U, U)$,

(4) $\bar{D}_{P}^{+}\left[\left(X_{1}, Y_{1}\right) \cup^{(2)}\left(X_{2}, Y_{2}\right)\right]=\bar{D}_{P}^{+}\left(X_{1}, Y_{1}\right) \cup^{(2)} \bar{D}_{P}^{+}\left(X_{2}, Y_{2}\right)$, $\bar{D}_{P}^{-}\left[\left(X_{1}, Y_{1}\right) \cup^{(2)}\left(X_{2}, Y_{2}\right)\right]=\bar{D}_{P}^{-}\left(X_{1}, Y_{1}\right) \cup^{(2)} \bar{D}_{P}^{-}\left(X_{2}, Y_{2}\right)$, 
(5) $\underline{D}_{P}^{+}\left[\left(X_{1}, Y_{1}\right) \cap^{(2)}\left(X_{2}, Y_{2}\right)\right]=\underline{D}_{P}^{+}\left(X_{1}, Y_{1}\right) \cap^{(2)} \underline{D}_{P}^{+}\left(X_{2}, Y_{2}\right)$, $\underline{D}_{P}^{-}\left[\left(X_{1}, Y_{1}\right) \cap^{(2)}\left(X_{2}, Y_{2}\right)\right]=\underline{D}_{P}^{-}\left(X_{1}, Y_{1}\right) \cap^{(2)} \underline{D}_{P}^{-}\left(X_{2}, Y_{2}\right)$,

(6) $\left(X_{1}, Y_{1}\right) \quad \subseteq^{(2)} \quad\left(X_{2}, Y_{2}\right) \quad \Rightarrow \quad \underline{D}_{P}^{+}\left(X_{1}, Y_{1}\right) \quad \subseteq^{(2)} \quad \underline{D}_{P}^{+}\left(X_{2}, Y_{2}\right) \quad$ and $\underline{D}_{P}^{-}\left(X_{1}, Y_{1}\right) \subseteq{ }^{(2)} \underline{D}_{P}^{-}\left(X_{2}, Y_{2}\right)$

(7) $\left(X_{1}, Y_{1}\right) \quad \subseteq^{(2)} \quad\left(X_{2}, Y_{2}\right) \quad \Rightarrow \quad \bar{D}_{P}^{-}\left(X_{1}, Y_{1}\right) \quad \subseteq^{(2)} \quad \bar{D}_{P}^{-}\left(X_{2}, Y_{2}\right) \quad$ and $\bar{D}_{P}^{+}\left(X_{1}, Y_{1}\right) \subseteq{ }^{(2)} \bar{D}_{P}^{+}\left(X_{2}, Y_{2}\right)$

(8) $\underline{D}_{P}^{+}\left[\left(X_{1}, Y_{1}\right) \cup(2)\left(X_{2}, Y_{2}\right)\right] \underline{\supseteq}^{(2)} \underline{D}_{P}^{+}\left(X_{1}, Y_{1}\right) \cup(2) \underline{D}_{P}^{+}\left(X_{2}, Y_{2}\right)$, $\underline{D}_{P}^{-}\left[\left(X_{1}, Y_{1}\right) \cup(2)\left(X_{2}, Y_{2}\right)\right] \supseteq^{(2)} \underline{D}_{P}^{-}\left(X_{1}, Y_{1}\right) \cup^{(2)} \underline{D}_{P}^{-}\left(X_{2}, Y_{2}\right)$,

(9) $\bar{D}_{P}^{+}\left[\left(X_{1}, Y_{1}\right) \cap^{(2)}\left(X_{2}, Y_{2}\right)\right] \subseteq^{(2)} \bar{D}_{P}^{+}\left(X_{1}, Y_{1}\right) \cap^{(2)} \bar{D}_{P}^{+}\left(X_{2}, Y_{2}\right)$, $\bar{D}_{P}^{-}\left[\left(X_{1}, Y_{1}\right) \cap^{(2)}\left(X_{2}, Y_{2}\right)\right] \subseteq^{(2)} \bar{D}_{P}^{-}\left(X_{1}, Y_{1}\right) \cap^{(2)} \bar{D}_{P}^{-}\left(X_{2}, Y_{2}\right)$,

(10) $\underline{D}_{P}^{+}\left[(U, U)-{ }^{(2)}(X, Y)\right]=(U, U)-{ }^{(2)} \bar{D}_{P}^{-}(X, Y)$, $\underline{D}_{P}^{-}\left[(U, U)-{ }^{(2)}(X, Y)\right]=(U, U)-{ }^{(2)} \bar{D}_{P}^{+}(X, Y)$,

(11) $\bar{D}_{P}^{+}\left[(U, U)-^{(2)}(X, Y)\right]=(U, U)-{ }^{(2)} \underline{D}_{P}^{-}(X, Y)$, $\bar{D}_{P}^{-}\left[(U, U)-{ }^{(2)}(X, Y)\right]=(U, U)-{ }^{(2)} \underline{D}_{P}^{+}(X, Y)$,

(12) $\underline{D}_{P}^{+}\left[\underline{D}_{P}^{+}(X, Y)\right]=\bar{D}_{P}^{+}\left[\underline{D}_{P}^{+}(X, Y)\right]=\underline{D}_{P}^{+}(X, Y)$, $\underline{D}_{P}^{-}\left[\underline{D}_{P}^{-}(X, Y)\right]=\bar{D}_{P}^{-}\left[\underline{D}_{P}^{-}(X, Y)\right]=\underline{D}_{P}^{-}(X, Y)$,

(13) $\bar{D}_{P}^{+}\left[\bar{D}_{P}^{+}(X, Y)\right]=\underline{D}_{P}^{+}\left[\bar{D}_{P}^{+}(X, Y)\right]=\bar{D}_{P}^{+}(X, Y)$, $\bar{D}_{P}^{-}\left[\bar{D}_{P}^{-}(X, Y)\right]=\underline{D}_{P}^{-}\left[\bar{D}_{P}^{-}(X, Y)\right]=\bar{D}_{P}^{-}(X, Y)$.

With respect to properties (10), (11) and (13), for operators $\underline{D}_{P}^{+}, \bar{D}_{P}^{+}, \underline{D}_{P}^{-}$and $\bar{D}_{P}^{-}$hold caveats related to abuse of notation analogous to the ones expressed for the corresponding properties of operators $\underline{I}_{P}^{\text {ortho }}$ and $\bar{I}_{P}^{\text {ortho }}$, and $\underline{I}_{P}^{\text {int }}$ and $\bar{I}_{P}^{\text {int }}$.

With respect to interval set $(W, Z) \in U^{\text {int }}$, the dominance-based rough approximations can be defined as follows:

$$
\begin{aligned}
& \underline{D}_{P}^{+i n t}(W, Z)=\left(\underline{D}_{P}^{+}(W), \bar{D}_{P}^{+}(Z),\right. \\
& \underline{D}_{P}^{-i n t}(W, Z)=\left(\underline{D}_{P}^{-}(W), \bar{D}_{P}^{-}(Z),\right. \\
& \bar{D}_{P}^{+i n t}(W, Z)=\left(\bar{D}_{P}^{+}(W), \underline{D}_{P}^{+}(Z),\right. \\
& \bar{D}_{P}^{-i n t}(W, Z)=\left(\bar{D}_{P}^{-}(W), \underline{D}_{P}^{-}(Z),\right.
\end{aligned}
$$

Taking into consideration the transformation function $\mathcal{T}$ converting orthopairs into equivalent interval sets, we have

$$
\begin{aligned}
\left.\underline{D}_{P}^{+i n t}(W, Z)\right) & \left.=\mathcal{T}\left(\underline{D}_{P}^{+}\left(\mathcal{T}^{-1}(W, Z)\right)\right), \underline{D}_{P}^{-i n t}(W, Z)\right)=\mathcal{T}\left(\underline{D}_{P}^{-}\left(\mathcal{T}^{-1}(W, Z)\right)\right), \\
\left.\bar{D}_{P}^{+i n t}(W, Z)\right) & \left.=\mathcal{T}\left(\bar{D}_{P}^{+}\left(\mathcal{T}^{-1}(W, Z)\right)\right), \bar{D}_{P}^{-i n t}(W, Z)\right)=\mathcal{T}\left(\bar{D}_{P}^{-}\left(\mathcal{T}^{-1}(W, Z)\right)\right) .
\end{aligned}
$$

Using operations $\cup^{\text {int }}, \cap^{\text {int }},{ }^{(2)}$ and inclusion $\subseteq^{\text {int }}$ on interval sets, we can express the properties of the dominance-based rough approximations of interval sets as follows. 
For any $(W, Z),\left(W_{1}, Z_{1}\right),\left(W_{2}, Z_{2}\right) \in U^{i n t}$ and for any $P \subseteq Q$,

(1) $\underline{D}_{P}^{+i n t}(W, Z) \subseteq \subseteq^{i n t}(W, Z) \subseteq \subseteq^{i n t} \bar{D}_{P}^{+i n t}(W, Z)$, $\underline{D}_{P}^{-i n t}(W, Z) \subseteq \subseteq^{i n t}(W, Z) \subseteq \subseteq^{i n t} \bar{D}_{P}^{-i n t}(W, Z)$,

(2) $\underline{D}_{P}^{+i n t}(\emptyset, \emptyset)=\bar{D}_{P}^{+i n t}(\emptyset, \emptyset)=(\emptyset, \emptyset), \underline{D}_{P}^{-i n t}(\emptyset, \emptyset)=\bar{D}_{P}^{-i n t}(\emptyset, \emptyset)=(\emptyset, \emptyset)$,

(3) $\underline{D}_{P}^{+i n t}(U, U)=\bar{D}_{P}^{+i n t}(U, U)=(U, U), \underline{D}_{P}^{-i n t}(U, U)=\bar{D}_{P}^{-i n t}(U, U)=(U, U)$,

(4) $\bar{D}_{P}^{+i n t}\left[\left(W_{1}, Z_{1}\right) \cup\right.$ int $\left.\left(W_{2}, Z_{2}\right)\right]=\bar{D}_{P}^{+i n t}\left(W_{1}, Z_{1}\right) \cup$ int $\bar{D}_{P}^{+i n t}\left(W_{2}, Z_{2}\right)$, $\bar{D}_{P}^{-i n t}\left[\left(W_{1}, Z_{1}\right) \cup i n t\left(W_{2}, Z_{2}\right)\right]=\bar{D}_{P}^{-i n t}\left(W_{1}, Z_{1}\right) \cup i n t \bar{D}_{P}^{-i n t}\left(W_{2}, Z_{2}\right)$,

(5) $\underline{D}_{P}^{+i n t}\left[\left(W_{1}, Z_{1}\right) \cap i n t\left(W_{2}, Z_{2}\right)\right]=\underline{D}_{P}^{+i n t}\left(W_{1}, Z_{1}\right) \cap^{i n t} \underline{D}_{P}^{+i n t}\left(W_{2}, Z_{2}\right)$, $\underline{D}_{P}^{-i n t}\left[\left(W_{1}, Z_{1}\right) \cap^{i n t}\left(W_{2}, Z_{2}\right)\right]=\underline{D}_{P}^{-i n t}\left(W_{1}, Z_{1}\right) \cap^{i n t} \underline{D}_{P}^{-i n t}\left(W_{2}, Z_{2}\right)$,

(6) $\left(W_{1}, Z_{1}\right) \subseteq{ }^{i n t}\left(W_{2}, Z_{2}\right) \Rightarrow \underline{D}_{P}^{+i n t}\left(W_{1}, Z_{1}\right) \subseteq{ }^{i n t} \underline{D}_{P}^{+i n t}\left(W_{2}, Z_{2}\right)$ and $\underline{D}_{P}^{-i n t}\left(W_{1}, Z_{1}\right) \subseteq{ }^{i n t} \underline{D}_{P}^{-i n t}\left(W_{2}, Z_{2}\right)$,

(7) $\left(W_{1}, Z_{1}\right) \subseteq{ }^{i n t}\left(W_{2}, Z_{2}\right) \Rightarrow \bar{D}_{P}^{-i n t}\left(W_{1}, Z_{1}\right) \subseteq^{i n t} \bar{D}_{P}^{-i n t}\left(W_{2}, Z_{2}\right)$ and $\bar{D}_{P}^{+i n t}\left(W_{1}, Z_{1}\right) \subseteq \subseteq^{i n t} \bar{D}_{P}^{+i n t}\left(W_{2}, Z_{2}\right)$

(8) $\underline{D}_{P}^{+i n t}\left[\left(W_{1}, Z_{1}\right) \cup\right.$ int $\left.\left(W_{2}, Z_{2}\right)\right] \supseteq^{i n t} \underline{D}_{P}^{+i n t}\left(W_{1}, Z_{1}\right) \cup \cup^{i n t} \underline{D}_{P}^{+i n t}\left(W_{2}, Z_{2}\right)$, $\underline{D}_{P}^{-i n t}\left[\left(W_{1}, Z_{1}\right) \cup^{i n t}\left(W_{2}, Z_{2}\right)\right] \supseteq^{i n t} \underline{D}_{P}^{-i n t}\left(W_{1}, Z_{1}\right) \cup{ }^{i n t} \underline{D}_{P}^{-i n t}\left(W_{2}, Z_{2}\right)$,

(9) $\bar{D}_{P}^{+i n t}\left[\left(W_{1}, Z_{1}\right) \cap i n t\left(W_{2}, Z_{2}\right) \subseteq^{i n t} \bar{D}_{P}^{+i n t}\left(W_{1}, Z_{1}\right) \cap^{i n t} \bar{D}_{P}^{+i n t}\left(W_{2}, Z_{2}\right)\right.$, $\bar{D}_{P}^{-i n t}\left[\left(W_{1}, Z_{1}\right) \cap i n t\left(W_{2}, Z_{2}\right) \subseteq^{i n t} \bar{D}_{P}^{-i n t}\left(W_{1}, Z_{1}\right) \cap^{i n t} \bar{D}_{P}^{-i n t}\left(W_{2}, Z_{2}\right)\right.$,

(10) $\underline{D}_{P}^{+i n t}\left[(U, U){ }^{(2)}(W, Z)\right]=(U, U)-\bar{D}_{P}^{-i n t}(W, Z)$, $\underline{D}_{P}^{-i n t}\left[(U, U)-{ }^{(2)}(W, Z)\right]=(U, U)-{ }^{(2)} \bar{D}_{P}^{+i n t}(W, Z)$,

(11) $\bar{D}_{P}^{+i n t}\left[(U, U)-^{(2)}(W, Z)\right]=(U, U)-\underline{D}_{P}^{-i n t}(W, Z)$, $\bar{D}_{P}^{-i n t}\left[(U, U)-^{(2)}(W, Z)\right]=(U, U)-\underline{D}_{P}^{+i n t}(W, Z)$,

(12) $\underline{D}_{P}^{+i n t}\left[\underline{D}_{P}^{+i n t}(W, Z)\right]=\bar{D}_{P}^{+i n t}\left[\underline{D}_{P}^{+i n t}(W, Z)\right]=\underline{D}_{P}^{+i n t}(W, Z)$, $\underline{D}_{P}^{-i n t}\left[\underline{D}_{P}^{-i n t}(W, Z)\right]=\bar{D}_{P}^{-i n t}\left[\underline{D}_{P}^{-i n t}(W, Z)\right]=\underline{D}_{P}^{-i n t}(W, Z)$,

(13) $\bar{D}_{P}^{+i n t}\left[\bar{D}_{P}^{+i n t}(W, Z)\right]=\underline{D}_{P}^{+i n t}\left[\bar{D}_{P}^{+i n t}(W, Z)\right]=\bar{D}_{P}^{+i n t}(W, Z)$, $\bar{D}_{P}^{-i n t}\left[\bar{D}_{P}^{-i n t}(W, Z)\right]=\underline{D}_{P}^{-i n t}\left[\bar{D}_{P}^{-i n t}(W, Z)\right]=\bar{D}_{P}^{-i n t}(W, Z)$.

Again, in properties (10), (11) and (13) of rough approximation operators $\underline{D}_{P}^{+i n t}$, $\bar{D}_{P}^{+i n t}, \underline{D}_{P}^{-i n t}$ and $\bar{D}_{P}^{-i n t}$, there are abuses of notation analogous to the ones expressed for the corresponding properties of the other rough approximation operators of orthopairs and interval sets considered previously.

In the context of the dominance-based approximations of the orthopairs, the reducts and the core can be defined as follows. Given $P \subseteq Q$ and $p \in P$, attribute $p$ is:

- superfluous in $P$ with respect to upward approximation of $X \subseteq U$ if $\underline{D}_{P}^{+}(X, Y)=\underline{D}_{P-\{p\}}^{+}(X, Y)$, which is equivalent to $\underline{D}_{P}^{-}(Y, X)=$ $\underline{D}_{P-\{p\}}^{-}(Y, X)$; otherwise, $p$ is upward indispensable in $P$;

- superfluous in $P$ with respect to downward approximation of $X \subseteq$ $U$ if $\underline{D}_{P}^{-}(X, Y)=\underline{D}_{P-\{p\}}^{-}(X, Y)$, which is equivalent to $\underline{D}_{P}^{+}(Y, X)=$ $\underline{D}_{P-\{p\}}^{+}(Y, X)$; otherwise, $p$ is downward indispensable in $P$. 
As in the other cases we have considered, with respect to the approximation of the orthopair $(X, Y) \in 3^{U}$,

- the set of all the upward indispensable attributes is the upward core, as well as the set of all the downward indispensable attributes is the downward core,

- any minimal subset $P \subseteq Q$, such that $\underline{D}_{P}^{+}(X, Y)=\underline{D}_{Q}^{+}(X, Y)$, is an upward reduct, as well as any minimal subset $P \subseteq Q$, such that $\underline{D}_{P}^{-}(X, Y)=\underline{D}_{Q}^{-}(X, Y)$, is called a downward reduct with respect to the approximation of $X$.

Taking into account the vague concept $(X, Y) \in 3^{U}$, we have to distinguish two cases:

- $X$ is positively correlated and $Y$ is negatively correlated with evaluations $v(x, q), q \in Q:$ in this case one can induce:

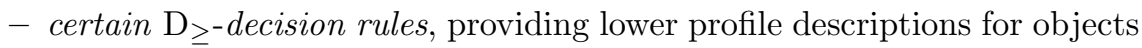
belonging to $\underline{D}_{Q}^{+}(X)$,

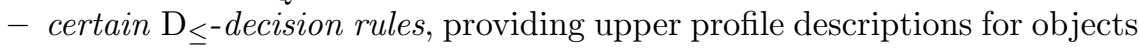
belonging to $\underline{D}_{Q}^{-}(Y)$,

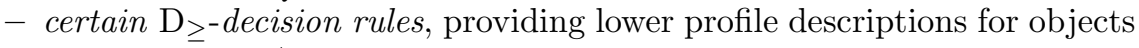
belonging to $\underline{D}_{Q}^{+}(U-Y)$,

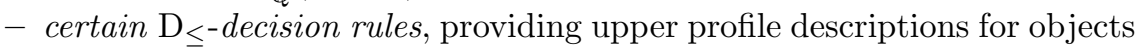
belonging to $\underline{D}_{Q}^{-}(U-X)$,

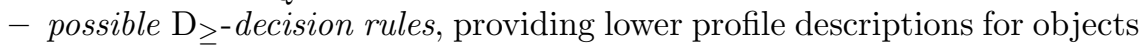
belonging to $\bar{D}_{Q}^{+}(X)$,

- possible $\mathrm{D}_{\leq-}$decision rules, providing upper profile descriptions for objects belonging to $\bar{D}_{Q}^{-}(Y)$,

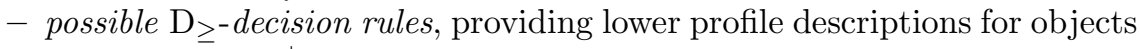
belonging to $\bar{D}_{Q}^{+}(U-Y)$,

- possible $\mathrm{D}_{\leq- \text {decision rules, providing upper profile descriptions for objects }}$ belonging to $\bar{D}_{Q}^{-}(U-X)$.

- $X$ is negatively correlated and $Y$ is positively correlated with evaluations $v(x, q), q \in Q$ : in this case one can induce:

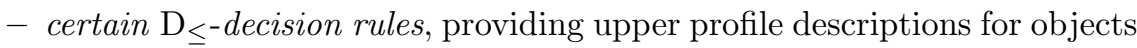
belonging to $\underline{D}_{Q}^{-}(X)$,

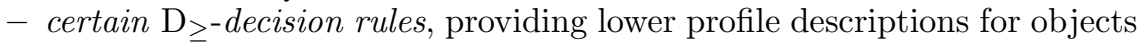
belonging to $\underline{D}_{Q}^{+}(Y)$,

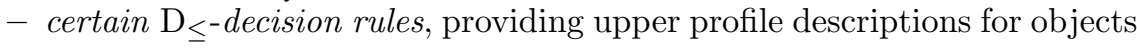
belonging to $\underline{D}_{Q}^{-}(U-Y)$,

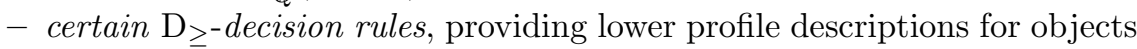
belonging to $\underline{D}_{Q}^{+}(U-X)$,

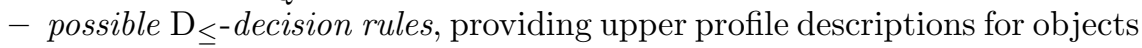
belonging to $\bar{D}_{Q}^{-}(X)$, 
- possible $\mathrm{D}_{\geq-}$decision rules, providing lower profile descriptions for objects belonging to $\bar{D}_{Q}^{+}(Y)$,

- possible $\mathrm{D}_{\leq- \text {decision rules, providing upper profile descriptions for objects }}$ belonging to $\bar{D}_{Q}^{-}(U-Y)$,

- possible $\mathrm{D}_{\geq- \text {decision rules, providing lower profile descriptions for objects }}$ belonging to $\bar{D}_{Q}^{+}(U-X)$.

\subsection{Didactic example}

Returning to the example introduced in subsection 2.1 and continued in subsections 3.1 and 4.1, the committee decided to apply DRSA on Table 2, so that imprecise information could be represented in terms of the rough approximations based on dominance. There is only one reduct that is also the core and it is composed of all the four subjects. The following certain decision rules were obtained:

rule 1"') if the evaluation on Mathematics is (at least) good, and the evaluation on Physics is at least (good), then the student is accepted, $\{S 1, S 2\}$,

rule 2"') if the evaluation on Mathematics is (at least) good, and the evaluation on Literature is at least (good), then the student is accepted, $\{S 2\}$,

rule 3"') if the evaluation on Physics is (at least) good, and the evaluation on Literature is at least (good), then the student is accepted, $\{S 2\}$,

rule 4"') if the evaluation on Mathematics is at least medium, and the evaluation on Physics is (at least) good, then the acceptance of the student is doubtful or the student is accepted, $\{S 1, S 2, S 3, S 12\}$,

rule 5"') if the evaluation on Mathematics is (at least) good, and the evaluation on Physics is at least medium, then the acceptance of the student is doubtful or the student is accepted, $\{S 1, S 2, S 8\}$,

rule 6"') if the evaluation on Mathematics is (at least) good, and the evaluation on Literature is (at least) good, then the acceptance of the student is doubtful or the student is accepted, $\{S 2\}$,

rule 7'") if the evaluation on Physics is (at least) good, and the evaluation on Literature is (at least) good, then the acceptance of the student is doubtful or the student is accepted, $\{S 2\}$,

rule 8"') if the evaluation on Mathematics is (at least) good, and the evaluation on Philosophy is (at least) good, then the acceptance of the student is doubtful or the student is accepted, $\{S 1, S 8\}$,

rule 9"') if the evaluation on Physics is (at least) good, and the evaluation on Philosophy is (at least) good, then the acceptance of the student is doubtful or the student is accepted, $\{S 1, S 3\}$,

rule 10"') if the evaluation on Mathematics is at most medium, then the acceptance of the student is doubtful or the student is rejected, 
$\{S 3, S 4, S 5, S 6, S 7, S 9, S 10, S 11, S 12, S 14, S 15\}$,

rule 11"') if the evaluation on Physics is at most medium, then the acceptance of the student is doubtful or the student is rejected,

$\{S 4, S 5, S 6, S 7, S 8, S 9, S 10, S 11, S 13, S 15\}$,

rule 12"') if the evaluation on Philosophy is (at most) bad, then the acceptance of the student is doubtful or the student is rejected, $\{S 4, S 12\}$,

rule 13"') if the evaluation on Literature is at most medium, and the evaluation on Philosophy is at most medium, then the acceptance of the student is doubtful or the student is rejected, $\{S 4, S 10, S 12, S 13, S 14\}$,

rule 14"') if the evaluation on Mathematics is (at most) bad, then the student is rejected, $\quad\{S 5, S 7, S 11, S 14, S 15\}$,

rule 15"') if the evaluation on Physics is (at most) bad, then the student is rejected, $\quad\{S 13, S 15\}$,

\section{Some extensions}

In this section we discuss some extensions of the rough approximation of the orthopairs we discussed in the previous sections. Let us start by considering the extension of the variable precision rough set approach. ${ }^{24}$

Given $P \subseteq Q$ and $X \subseteq U$, using the $P$-elementary sets, the $\alpha$ variable precision $P$-lower approximation and the $\beta P$-upper approximation of $X, 0 \leq \beta<\alpha \leq 1$, are defined, respectively, as follows:

$$
\begin{aligned}
& \underline{I}_{P}^{\alpha}(X)=\bigcup_{x \in U: \mu_{X}^{P}(x) \geq \alpha} I_{P}(x), \\
& \bar{I}_{P}^{\beta}(X)=\bigcup_{x \in U: \mu_{X}^{P}(x)>\beta} I_{P}(x) .
\end{aligned}
$$

Given subset $P \subseteq Q$ and orthopair $(X, Y) \in 3^{U}$, using the $P$-elementary sets, the $\left(\alpha_{P O S}, \alpha_{N E G}, \beta_{P O S}, \beta_{N E G}\right)$ variable precision $P$-lower and the $P$-upper approximations of $(X, Y)$, with

$$
0 \leq \beta_{P O S}<\alpha_{P O S} \leq 1, \quad 0 \leq \beta_{N E G}<\alpha_{N E G} \leq 1,
$$

are defined, respectively, as follows:

$$
\begin{aligned}
& \underline{I}_{P}^{\alpha_{P O S}, \alpha_{N E G}}(X, Y)=\left(\underline{I}_{P}^{\alpha_{P O S}}(X), \underline{I}_{P}^{\alpha_{N E G}}(Y)\right), \\
& \bar{I}_{P}^{\beta_{P O S}, \beta_{N E G}}(X, Y)=\left(\bar{I}_{P}^{\beta_{P O S}}(X), \bar{I}_{P}^{\beta_{N E G}}(Y)\right) .
\end{aligned}
$$

Analogous definition can be given within the dominance-based rough set approach $^{25}$ (see also ${ }^{26}$ ). Given $P \subseteq Q$ and $X \subseteq U$, let us define first the upward rough membership $\mu_{X}^{P+}(x)$ and the downward rough membership $\mu_{X}^{P-}(x)$ :

$$
\mu_{X}^{P+}(x)=\frac{\left|D_{P}^{+}(x) \cap X\right|}{\left|D_{P}^{+}(x)\right|},
$$


and

$$
\mu_{X}^{P-}(x)=\frac{\left|D_{P}^{-}(x) \cap X\right|}{\left|D_{P}^{-}(x)\right|} .
$$

Using the dominance-based rough memberships $\mu_{X}^{P+}(x)$ and $\mu_{X}^{P-}(x)$, and fixing

$$
0 \leq \beta<\alpha \leq 1,
$$

the dominance-based rough set approximations of $X \subseteq U$ with respect to $P \subseteq Q$ can be defined as follows:

- the upward $\alpha$ variable precision $P$-lower approximation $\underline{D}_{P}^{\alpha+}(X)$ and the upward $\beta$ variable precision $P$-upper approximation $\bar{D}_{P}^{\beta+}(X)$,

$$
\begin{aligned}
& \underline{D}_{P}^{\alpha+}(X)=\bigcup_{x \in U: \mu_{X}^{P+}(x) \geq \alpha} D_{P}^{+}(x), \\
& \bar{D}_{P}^{\beta+}(X)=\bigcup_{x \in U: \mu_{X}^{P+}(x)>\beta} D_{P}^{+}(x) .
\end{aligned}
$$

- the downward $\alpha$ variable precision $P$-lower approximation $\underline{D}_{P}^{\alpha-}(X)$ and the downward $\beta$ variable precision $P$-upper approximation $\bar{D}_{P}^{\beta-}(X)$,

$$
\begin{aligned}
& \underline{D}_{P}^{\alpha-}(X)=\bigcup_{x \in U: \mu_{X}^{P-}(x) \geq \alpha} D_{P}^{-}(x), \\
& \bar{D}_{P}^{\beta-}(X)=\bigcup_{x \in U: \mu_{X}^{P-}(x)>\beta} D_{P}^{-}(x) .
\end{aligned}
$$

Given $P \subseteq Q$ and $\alpha_{P O S}, \alpha_{N E G}, \beta_{P O S}$ and $\beta_{N E G}$ such that

$$
0 \leq \beta_{P O S}<\alpha_{P O S} \leq 1,0 \leq \beta_{N E G}<\alpha_{N E G} \leq 1,
$$

variable precision dominance-based rough approximations of $(X, Y) \in 3^{U}$ can be defined as follows:

- upward $\left(\alpha_{P O S}, \alpha_{N E G}\right)$ variable precision $P$-lower approximation

$$
\underline{D}_{P}^{\alpha_{P O S}, \alpha_{N E G}+}(X, Y)=\left(\underline{D}_{P}^{\alpha_{P O S}+}(X), \underline{D}_{P}^{\alpha_{N E G}-}(Y)\right),
$$

- upward $\left(\beta_{P O S}, \beta_{N E G}\right)$ variable precision $P$-upper approximation

$$
\bar{D}_{P}^{\beta_{P O S}, \beta_{N E G}+}(X, Y)=\left(\bar{D}_{P}^{\beta_{P O S}+}(X), \bar{D}_{P}^{\beta_{N E G}{ }^{-}}(Y)\right),
$$

- downward $\left(\alpha_{P O S}, \alpha_{N E G}\right)$ variable precision $P$-lower approximation

$$
\underline{D}_{P}^{\alpha_{P O S}, \alpha_{N E G^{-}}}(X, Y)=\left(\underline{D}_{P}^{\alpha_{P O S^{-}}}(X), \underline{D}_{P}^{\alpha_{N E G^{+}}}(Y)\right),
$$

- downward $\left(\beta_{P O S}, \beta_{N E G}\right)$ variable precision $P$-upper approximation

$$
\bar{D}_{P}^{\beta_{P O S}, \beta_{N E G}-}(X, Y)=\left(\bar{D}_{P}^{\beta_{P O S^{-}}}(X), \bar{D}_{P}^{\beta_{N E G}+}(Y)\right) \text {. }
$$


Fuzzy set theory permits to extend in a natural way the idea of rough approximations of the orthopairs. Let us remember that a fuzzy set $X$ in a universe of discourse $U$ is identified by its membership function $\mu_{X}: U \rightarrow[0,1]$, such that, for each $y \in U, \mu_{X}(y)$ represents the credibility with which $y$ belongs to the set $X$. To extend rough approximations to the context of fuzzy set theory, we need some concepts and operators of fuzzy logic. For each proposition $p$, we consider its truth value $v(p)$, ranging from $v(p)=0$ ( $p$ is definitely false) to $v(p)=1$ ( $p$ is definitely true), and for all intermediate values, the greater $v(p)$, the more credible is the truth of $p$. The following fuzzy logic connectives can be considered

- the negation, being a non-increasing function $N:[0,1] \rightarrow[0,1]$ for which $N(0)=1$ and $N(1)=0$, such that, given proposition $p, N(v(p))$ states the credibility of the negation of $p$;

- the t-norm $T$ and the t-conorm $T^{*}$, being two functions $T:[0,1] \times[0,1] \rightarrow[0,1]$ and $T^{*}:[0,1] \times[0,1] \rightarrow[0,1]$, such that, given two propositions, $p$ and $q, T(v(p), v(q))$ represents the credibility of the conjunction of $p$ and $q$, and $T^{*}(v(p), v(q))$ represents the credibility of the disjunction of $p$ and $q$. t-norm $T$ and t-conorm $T^{*}$ must satisfy the following properties:

$$
\begin{gathered}
T(\alpha, \beta)=T(\beta, \alpha) \text { and } T^{*}(\alpha, \beta)=T^{*}(\beta, \alpha), \text { for all } \alpha, \beta \in[0,1], \\
T(\alpha, \beta) \leq T(\gamma, \delta) \text { and } T^{*}(\alpha, \beta) \leq T^{*}(\gamma, \delta) \text { for all } \alpha, \beta, \gamma, \delta \in[0,1] \\
\text { such that } \alpha \leq \gamma \text { and } \beta \leq \delta, \\
T(\alpha, T(\beta, \gamma))=T(T(\alpha, \beta), \gamma) \text { and } T^{*}\left(\alpha, T^{*}(\beta, \gamma)\right)=T^{*}\left(T^{*}(\alpha, \beta), \gamma\right), \\
\text { for all } \alpha, \beta, \gamma \in[0,1], \\
T(1, \alpha)=\alpha \text { and } T^{*}(0, \alpha)=\alpha, \text { for all } \alpha \in[0,1] .
\end{gathered}
$$

- the fuzzy implication, being a function $I:[0,1] \times[0,1] \rightarrow[0,1]$, such that, given two propositions, $p$ and $q, I(v(p), v(q))$ represents the credibility of the implication of $q$ by $p$. A fuzzy implication must satisfy the following properties: $^{27}$

$$
\begin{gathered}
I(\alpha, \beta) \geq I(\gamma, \beta) \text { for all } \alpha, \beta, \gamma \in[0,1], \text { such that } \alpha \leq \gamma \\
I(\alpha, \beta) \geq I(\alpha, \gamma) \text { for all } \alpha, \beta, \gamma \in[0,1], \text { such that } \beta \geq \gamma \\
I(0, \alpha)=1, I(\alpha, 1)=1 \text { for all } \alpha \in[0,1] \\
I(1,0)=0 .
\end{gathered}
$$

Typical fuzzy connectives are: 
- negation: $N(x)=1-x$

- t-norm: $T(x, y)=\min (x, y)$;

- t-conorm: $T^{*}(x, y)=\max (x, y)$

- implication: $I(x, y)=\max (1-x, y)$.

A fuzzy binary relation $R$ is a function $R: U \times U \rightarrow[0,1]$ such that for all $x, y \in$ $U, R(x, y)$ represents the fuzzy membership of $(x, y)$ to the relations $R$. Several properties can be considered for fuzzy binary relations. In the following, we shall consider only two:

- reflexivity: a fuzzy binary relation $R$ is reflexive if for all $x \in U, R(x, x)=1$;

- t-transitivity: a fuzzy binary relation $R$ is transitive with respect to the t-norm $T$ if, for all $x, y, z \in U, T(R(x, y), R(y, z)) \leq R(x, z)$.

A definition of fuzzy rough approximation has been proposed by Radzikowska and Kerre ${ }^{5}$ on a previous idea of Dubois and Prade, ${ }^{4}$ that, considering a reflexive fuzzy binary relation $R$, defined the lower approximation $\underline{R}(X, x)$ and the upper approximation $\bar{R}(X, x))$ of $X$ as follows:

$$
\begin{aligned}
& \underline{R}(X, x))=\inf _{y \in U}\left(I\left(R(x, y), \mu_{X}(y)\right)\right), \\
& \bar{R}(X, x))=\sup _{y \in U}\left(T\left(R(x, y), \mu_{X}(y)\right)\right) .
\end{aligned}
$$

Greco, Matarazzo and Słowiński ${ }^{28-30}$ proposed a slightly more general definition:

$$
\begin{aligned}
& \underline{R}^{\prime}(X, x)=T_{y \in U}\left(I\left(R(x, y), \mu_{X}(y)\right)\right), \\
& \bar{R}^{\prime}(X, x)=T_{y \in U}^{*}\left(T\left(R(x, y), \mu_{X}(y)\right)\right) .
\end{aligned}
$$

Observe that

$$
\underline{R}(X, x)=\underline{R}^{\prime}(X, x)
$$

in case $T(x, y)=\min (x, y)$, as well as,

$$
\bar{R}(X, x)=\bar{R}^{\prime}(X, x)
$$

in case $T^{*}(x, y)=\max (x, y)$.

Intuitionistic fuzzy sets ${ }^{31}$ can be considered as a fuzzy counterpart of the orthopairs (see, e.g. ${ }^{32}$ ). An intuitionistic fuzzy set $X$ in a universe of discourse $U$ is identified by a fuzzy membership $\mu_{X}: U \rightarrow[0,1]$ and a fuzzy non-membership function $\nu_{X}: U \rightarrow[0,1], \mu_{X}(y)+\nu_{X}(y) \leq 1$ for all $y \in U$, with $\mu_{X}(y)$ being the degree of membership and $\nu_{X}(y)$ being the degree of non-membership of $y$ to $X$. Given a fuzzy similarity relation $R$ in $U$, the lower approximation $\underline{R}(X)$ and the upper approximation $\bar{R}(X)$ of the intuitionistic fuzzy set $X$ can be defined by assigning fuzzy rough approximation of membership $\mu_{X}(x)$ and fuzzy rough approximation of non-membership $\nu_{X}(x)$ to each $x \in U$, so that, taking into account the Radzikowska and Kerre's rough approximations, we get 
- the lower approximation of the membership of $x$ to $X$

$$
\underline{R}^{+}(X, x)=i n f_{y \in U}\left(I\left(R(x, y), \mu_{X}(y)\right)\right),
$$

- the upper approximation of the membership of $x$ to $X$

$$
\bar{R}^{+}(X, x)=\sup _{y \in U}\left(T\left(R(x, y), \mu_{X}(y)\right)\right),
$$

- the lower approximation of the non-membership of $x$ to $X$

$$
\underline{R}^{-}(X, x)=i n f_{y \in U}\left(I\left(R(x, y), \nu_{X}(y)\right)\right),
$$

- the upper approximation of the non-membership of $x$ to $X$

$$
\bar{R}^{-}(X, x)=\sup _{y \in U}\left(T\left(R(x, y), \nu_{X}(y)\right)\right) .
$$

On the other hand, taking into account the rough approximations proposed by Greco, Matarazzo and Słowiński, we get

- the lower approximation of the membership of $x$ to $X$

$$
\underline{R}^{+}(X, x)=T_{y \in U}\left(I\left(R(x, y), \mu_{X}(y)\right)\right),
$$

- the upper approximation of the membership of $x$ to $X$

$$
\bar{R}^{+}(X, x)=T_{y \in U}^{*}\left(T\left(R(x, y), \mu_{X}(y)\right)\right),
$$

- the lower approximation of the non-membership of $x$ to $X$

$$
\underline{R}^{-}(X, x)=T_{y \in U}\left(I\left(R(x, y), \nu_{X}(y)\right)\right),
$$

- the upper approximation of the non-membership of $x$ to $X$

$$
\bar{R}^{-}(X, x)=T_{y \in U}^{*}\left(T\left(R(x, y), \nu_{X}(y)\right)\right) .
$$

The rough approximation of an intuitionistic fuzzy set according to the Radzikowska and Kerre's definition has been proposed in. ${ }^{33}$ The rough approximation of an intuitionistic fuzzy set according to the Greco, Matarazzo and Słowiński's definition is new.

One can also consider a fuzzy DRSA approximation of an intuitionistic fuzzy set. With this aim, let us recall the DRSA fuzzy approximations. In this case, since the dominance relations with which we we want to approximate fuzzy sets are preorders, we have to consider a fuzzy binary relation $R$ on $U$, being a fuzzy preorder, that is, $R$ has to be reflexive and transitive. ${ }^{27}$ Also in this case, there exist two main definitions:

- the fuzzy dominance-based approximation proposed by Greco, Inuiguchi and Słowiński, ${ }^{34}$ in the following referred to as GIS DRSA approximation, which assigns to each $x \in U$

- the upward lower approximation membership

$$
\left.\underline{R}^{\uparrow}(X, x)\right)=i n f_{y \in U}\left(I\left(R(y, x), \mu_{X}(y)\right)\right),
$$


- the upward upper approximation membership

$$
\left.\bar{R}^{\uparrow}(X, x)\right)=\sup _{y \in U}\left(T\left(R(x, y), \mu_{X}(y)\right)\right),
$$

- the downward lower approximation membership

$$
\left.\underline{R}^{\downarrow}(X, x)\right)=i n f_{y \in U}\left(I\left(R(x, y), \mu_{X}(y)\right)\right),
$$

- the downward upper approximation membership

$$
\left.\bar{R}^{\downarrow}(X, x)\right)=\sup _{y \in U}\left(T\left(R(y, x), \mu_{X}(y)\right)\right) ;
$$

- the fuzzy dominance-based approximation proposed by Greco, Matarazzo and Słowiński, ${ }^{29,35}$ in the following referred to as GMS DRSA approximation, which assigns to each $x \in U$

- the upward lower approximation membership

$$
\left.\underline{R}^{\uparrow \prime}(X, x)\right)=T_{y \in U}\left(I\left(R(y, x), \mu_{X}(y)\right)\right),
$$

- the upward upper approximation membership

$$
\left.\bar{R}^{\uparrow \prime}(X, x)\right)=T_{y \in U}^{*}\left(T\left(R(x, y), \mu_{X}(y)\right)\right),
$$

- the downward lower approximation membership

$$
\left.\underline{R}^{\downarrow^{\prime}}(X, x)\right)=T_{y \in U}\left(I\left(R(x, y), \mu_{X}(y)\right)\right),
$$

- the downward upper approximation membership

$$
\left.\bar{R}^{\downarrow^{\prime}}(X, x)\right)=T_{y \in U}^{*}\left(T\left(R(y, x), \mu_{X}(y)\right)\right) .
$$

Taking into account the above fuzzy dominance-based rough approximations we can define the following fuzzy dominance-based rough approximations of an intuitionistic fuzzy set $X$ on $U$, identified by the membership function $\mu_{X}$ and the non-membership function $\nu_{X}$ :

- adopting the GIS DRSA approximation, we have

- the upward lower approximation of the membership of $x$ to $X$

$$
\left.\underline{R}^{\uparrow}(X, x)\right)=i n f_{y \in U}\left(I\left(R(y, x), \mu_{X}(y)\right)\right),
$$

- the upward upper approximation of the membership of $x$ to $X$

$$
\left.\bar{R}^{\uparrow}(X, x)\right)=\sup _{y \in U}\left(T\left(R(x, y), \mu_{X}(y)\right)\right),
$$

- the downward lower approximation of the non-membership of $x$ to $X$

$$
\left.\underline{R}^{\downarrow}(X, x)\right)=i n f_{y \in U}\left(I\left(R(x, y), \nu_{X}(y)\right)\right),
$$

- the downward upper approximation of the non-membership of $x$ to $X$

$$
\left.\bar{R}^{\downarrow}(X, x)\right)=\sup _{y \in U}\left(T\left(R(y, x), \nu_{X}(y)\right)\right) ;
$$

- adopting the GIS DRSA approximation we have 
- the upward lower approximation of the membership of $x$ to $X$

$$
\left.\underline{R}^{\uparrow}(X, x)\right)=T_{y \in U}\left(I\left(R(y, x), \mu_{X}(y)\right)\right),
$$

- the upward upper approximation of the membership of $x$ to $X$

$$
\left.\bar{R}^{\uparrow \prime}(X, x)\right)=T_{y \in U}^{*}\left(T\left(R(x, y), \mu_{X}(y)\right)\right),
$$

- the downward lower approximation of the non-membership of $x$ to $X$

$$
\left.\underline{R}^{\downarrow^{\prime}}(X, x)\right)=T_{y \in U}\left(I\left(R(x, y), \nu_{X}(y)\right)\right),
$$

- the downward upper approximation of the non-membership of $x$ to $X$

$$
\left.\bar{R}^{\downarrow^{\prime}}(X, x)\right)=T_{y \in U}^{*}\left(T\left(R(y, x), \nu_{X}(y)\right)\right) .
$$

The GIS DRSA approximation, as well as the GMS DRSA approximation, are new and different from the DRSA rough approximation of an intuitionistic fuzzy set proposed on another basis in. ${ }^{36}$

\section{Conclusions}

We proposed a new rough set approach to representation of imperfect knowledge that permits to distinguish between vagueness, due to 'a priori' imprecise knowledge, and ambiguity, due to 'a posteriori' approximation based on granular information. This approach consists in the rough approximation of imprecise knowledge represented by an orthopair which assigns to a given concept two sets of objects in the universe of discourse: the set of positive objects that for sure belong to the considered concept, and the set of negative objects that for sure do not belong to the considered concept. To each orthopair we associate, moreover, other two pairs of sets of objects:

- the pair composed of the lower approximations of the positive set and the negative set,

- the pair composed of the upper approximations of the positive set and the negative set.

We considered several types of rough approximations:

- rough approximations based on indiscernibility relation,

- rough approximations based on dominance relation,

- rough approximations of fuzzy orthopairs (identical with intuitionistic fuzzy sets) based on fuzzy relation,

- variable precision rough approximations accepting some tolerance for positive and negative sets.

We believe that the proposed distinction between vagueness and ambiguity in view of handling these two faces of imperfect knowledge in a joint framework has a great potential in real life applications. Moreover, our approach is based on a new 
algebraic model of rough set theory that extends the Brouwer-Zadeh lattice ${ }^{14}$ by introducing a new operator called Pawlak operator. ${ }^{18,20}$ In this way, we obtain an algebraic model which permits a joint consideration of vagueness and uncertainty, typical of fuzzy set theory, with ambiguity related to indiscernibility and coarseness, typical of rough set theory. We plan to investigate more in detail the properties of this new algebraic model and we are confident that practical applications will also benefit from these studies.

\section{References}

1. Pawlak, Z., Rough Sets. International Journal of Computer and Information Sciences, 11 (1982) 341-356.

2. Pawlak, Z., Rough Sets. Kluwer, Dordrecht, 1991.

3. Dubois, D. and Prade, H., Foreword. In: Z. Pawlak, Rough Sets. Kluwer, Dordrecht, 1991.

4. Dubois, D. and Prade, H., Putting rough sets and fuzzy sets together. In: R. Słowiński (ed.), Intelligent Decision Support: Handbook of Applications and Advances of the Rough Sets Theory, Kluwer, Dordrecht, 1992, pp. 203-232.

5. Radzikowska, A.M. and Kerre, E.E., A comparative study of fuzzy rough sets. Fuzzy Sets and systems, 126 (2002) 137-155.

6. Frege, G., Grundlagen der Arithmetik 2. Verlag von Herman Pohle, Jena, 1893.

7. Pawlak, Z., Vagueness - a rough set view. In: Structures in logic and computer science, Springer, Berlin, Heidelberg, 1997, pp. 106-117.

8. Pawlak, Z., A treatise on rough sets. In: Transactions on rough sets $I V$, Springer, Berlin, 2005, pp. 1-17.

9. Ciucci, D., Orthopairs and granular computing. Granular Computing, 1 (2016) 159170.

10. Monteiro, A., Sur les Algèbres de Heyting symétriques. Portugaliae Mathematica, 39 (1980) 1-237.

11. Pedrycz, W., Shadowed sets: representing and processing fuzzy sets. IEEE Transactions on Systems, Man, and Cybernetics, Part B (Cybernetics), 28 (1998) 103-109.

12. Cattaneo, G., Dalla Chiara, M., Giuntini, R. and Paoli, F., Quantum logic and nonclassical logics. In: Engesser, K., Gabbay, D. M., Lehmann, D. (Eds.), Handbook of Quantum Logic and Quantum Structures. Elsevier, Amsterdam, 2009, pp. 127-226.

13. Yao, Y.Y., Interval-set algebra for qualitative knowledge representation. Proceedings of the Fifth International Conference on Computing and Information, 1993, pp. 370374 .

14. Cattaneo, G. and Nisticò, G., Brouwer-Zadeh poset and three-valued Łukasiewicz posets. Fuzzy Sets and Systems, 33 (1989) 165-190.

15. Greco, S., Matarazzo, B. and Słowiński, R., Rough set theory for multicriteria decision analysis. European Journal of Operational Research, 129 (2001) 1-47.

16. Greco, S., Matarazzo, B. and Słowiński, R., Rough sets methodology for sorting problems in presence of multiple attributes and criteria. European Journal of Operational Research, 138 (2002) 247-259.

17. Greco, S., Matarazzo, B., Słowiński, R., Rough approximation by dominance relations. International Journal of Intelligent Systems, 17 (2002) 153-171.

18. Greco, S., Matarazzo, B. and Słowiński, R., Distinguishing vagueness from ambiguity by means of Pawlak-Brouwer-Zadeh lattices. In: S. Greco et al. (eds), International Conference on Information Processing and Management of Uncertainty in Knowledge- 
Based Systems, Springer, Berlin, 2012, pp. 624-632.

19. Polkowski, L., Rough Sets. Physica-Verlag, Heidelberg, 2002.

20. Greco, S., Matarazzo, B. and Słowiński, R., Distinguishing Vagueness from Ambiguity in Dominance-Based Rough Set Approach by Means of a Bipolar Pawlak-BrouwerZadeh Lattice. In: International Joint Conference on Rough Sets. Springer, Cham, 2017, pp. 81-93.

21. Słowiński, R., Greco, S. and Matarazzo, B., Rough-Set-Based Decision Support. Chapter 19 in: E.K. Burke and G. Kendall (eds.), Search Methodologies: Introductory Tutorials in Optimization and Decision Support Techniques, 2nd edition, Springer, New York, 2014, pp. 557-609.

22. Greco, S., Matarazzo, B., Słowiński, R., The Bipolar Complemented de Morgan Brouwer-Zadeh Distributive Lattice as an Algebraic Structure for the Dominancebased Rough Set Approach. Fundamenta Informaticae, 115, (2012) 25-56.

23. Susmaga, R., Słowiński, R., Greco, S. and Matarazzo, B., Generation of reducts and rules in multi-attribute and multi-criteria classification. Control and Cybernetics, 29(4) (2000) 969-988.

24. Ziarko, W., Variable precision rough set model. Journal of computer and system sciences, 46(1) (1993) 39-59.

25. Greco, S., Matarazzo, B., Słowiński, R. and Stefanowski, J., Variable consistency model of dominance-based rough set approach. In: W. Ziarko and Y. Yao (eds.), LNAI 2005, Rough Sets and Current Trends in Computing, Springer-Verlag, Berlin, 2000, pp. 170-181.

26. Błaszczyński, J., Greco, S., Słowiński, R. and Szelạg, M., Monotonic variable consistency rough set approaches. International Journal of Approximate Reasoning, 50 (2009) 979-999

27. Fodor, J. and Roubens, M., Fuzzy Preference Modelling and Multicriteria Decision Support. Kluwer, Dordrecht, 1994.

28. Greco, S., Matarazzo, B. and Słowiński, R., Fuzzy similarity relation as a basis for rough approximations. In: International Conference on Rough Sets and Current Trends in Computing. Springer, Berlin, 1998, pp. 283-289.

29. Greco, S., Matarazzo, B. and Słowiński, R., The use of rough sets and fuzzy sets in MCDM. Chapter 14 in: T. Gal, T. Stewart, T. Hanne (eds.), Advances in Multiple Criteria Decision Making, Kluwer Academic Publishers, Boston, 1999, pp. 14.1-14.59.

30. Greco, S., Matarazzo, B. and Słowiński, R., Rough set processing of vague information using fuzzy similarity relations. In: C. Calude and G. Paun (eds.), From Finite to Infinite, Springer, Berlin, 2000, pp. 149-173.

31. Atanassov, K.T., Intuitionistic fuzzy sets. Fuzzy Sets and Systems, 20 (1986) 87-96.

32. Coker, D., A note on intuitionistic sets and intuitionistic points. Turkish Journal of Mathematics, 20 (1996) 343-351.

33. Cornelis, C., Cock, M.D. and Kerre, E.E., Intuitionistic fuzzy rough sets: at the crossroads of imperfect knowledge. Expert Systems, 20 (2003) 260-270.

34. Greco, S., Inuiguchi, M. and Słowiński, R., Dominance-based rough set approach using possibility and necessity measures. In: J.J. Alpigini, J.F. Peters, A. Skowron, N. Zhong (eds.), Rough Sets and Current Trends in Computing, LNAI 2475, Springer, Berlin, 2002, pp. 85-92.

35. Greco, S., Matarazzo, B. and Słowiński, R., A fuzzy extension of the rough set approach to multicriteria and multiattribute sorting. In: J. Fodor, B. De Baets and P. Perny (eds.), Preferences and Decisions under Incomplete Information, PhysicaVerlag, Heidelberg, 2000, pp. 131-154.

36. Huang, B., Zhuang, Y.L., Li, H.X. and Wei, D.K., A dominance intuitionistic fuzzy- 
rough set approach and its applications. Applied Mathematical Modelling, 37 (2013) 7128-7141. 